\title{
DYNAMIC AND STEADY-STATE BEHAVIOR OF CONTINUOUS SEDIMENTATION*
}

\author{
STEFAN DIEHL ${ }^{\dagger}$
}

\begin{abstract}
Continuous sedimentation of solid particles in a liquid takes place in a clarifierthickener unit, which has one feed inlet and two outlets. The process can be modeled by a nonlinear scalar conservation law with point source and discontinuous flux function. This paper presents existence and uniqueness results in the case of varying cross-sectional area and a complete classification of the steady-state solutions when the cross-sectional area decreases with depth. The classification is utilized to formulate a static control strategy for the large discontinuity called the sludge blanket that appears in steady-state operation. A numerical algorithm and a few simulations are also presented.
\end{abstract}

Key words. conservation laws, discontinuous flux, point source, continuous sedimentation, clarifier-thickener, settler

AMS subject classifications. 35L65, 35Q80, 35R05

PII. S0036139995290101

1. Introduction. Continuous sedimentation of solid particles takes place in a liquid in a clarifier-thickener unit (or settler); see Fig. 2.1. Such a process is used, for example, in waste water treatment and in the chemical and mineral industries. The purpose is to provide a clear liquid at the top and a high concentration of solids at the bottom. Discontinuities in the concentration profile are observed in reality and under normal operating conditions there is a large discontinuity in the thickening zone called the sludge blanket.

Previous works. Previous studies of the clarifier-thickener unit have usually been confined to the modeling of the thickening zone with emphasis on the sludge blanket and the prediction of the underflow concentration; see [2]-[6], [14], [16]-[19], [33], [36]. Dynamic models of the entire clarifier-thickener unit mostly have been presented as simulation models, usually in the waste water research field. Some recent references of one-dimensional models are [16], [21], [35], [37], [38]. Because of the nonlinear phenomena of the continuous sedimentation process, it is difficult to classify the steady-state solutions for different values of the feed concentration and the volume flows; see [7], [29], [30], [34]. Particularly interesting results are presented by Chancelier, de Lara, and Pacard [7]. They introduce a good mathematical definition of the often-used term limiting flux, the maximum mass-flux capacity of the thickening zone at steady state. Their main result is a classification of the steady-state behavior of a settler with decreasing cross-sectional area with respect to the limiting flux. When the settler is fed with a mass flux greater than the limiting flux, it becomes overloaded, which means that the effluent at the top is not clear water. They also show that any steady-state solution has at most one discontinuity in the clarification zone. Solutions in the thickening zone are described only qualitatively, because of a general assumption on the constitutive settling flux function.

*Received by the editors August 9, 1995; accepted for publication (in revised form) April 30, 1996. This research was supported in part by the Royal Swedish Academy of Sciences.

http://www.siam.org/journals/siap/57-4/29010.html

${ }^{\dagger}$ Department of Mathematics, Lund Institute of Technology, P.O. Box 118, S-221 00 Lund, Sweden (diehl@maths.lth.se). 
In [11], the author presented a dynamic model of a settler with constant crosssectional area, including the prediction of the effluent and underflow concentrations. Construction of solutions and a proof of uniqueness were obtained by using the method of characteristics and a generalized entropy condition according to the theory in [10]. The different steady-state solutions were also presented explicitly. In [9], analysis of the sedimentation of multicomponent particles is presented. The results of [9], [11] have been used for an implementation of the settler model within a simulation model of a waste water treatment plant; see [13]. Comparisons with other models are presented in [25], [26].

The basic model equation for the sedimentation in the thickening zone used in almost all the references above is a scalar conservation law of the form $u_{t}+f(u)_{x}=0$. It is well known that the entropy condition by Oleinik [32] guarantees a unique, physically relevant solution with stable discontinuities. The equivalence between the entropy condition and the so-called viscous profile condition, where the unique solution is obtained by adding a small diffusion or viscosity term to the conservation law, is well established; see, e.g., [22], [27].

When it comes to the modeling of the entire settler including the feed inlet and the outlets, a number of ad hoc assumptions have been presented in the literature. To avoid such assumptions, a generalized entropy condition, condition $\Gamma$, was presented in [10], and it is the key behind the results in [11] and in the present paper. This condition is used to establish the unique connection between the concentration of the feed inlet with the concentrations in the settler just above and below the feed point and the connection between the outlet concentrations and the concentrations at the top and the bottom of the settler. The equivalence between condition $\Gamma$ and the viscous profile condition is presented in [12]. The stability of the viscous profiles is analyzed in [15].

Contents. In section 2 we describe the clarifier-thickener unit and the basic constitutive assumption, by Kynch [28], used in the modeling of sedimentation: the flux of particles per unit area and time is a function of the concentration only. Hence, there is no modeling of effects such as compression or diffusion. The conservation of mass can be used to obtain the scalar conservation law

$$
A(x) \frac{\partial u}{\partial t}+\frac{\partial}{\partial x}(A(x) F(u, x))=S(t) \delta(x),
$$

where $u=u(x, t)$ is the concentration, $\delta$ is the Dirac measure, $S$ is a source term modeling the feed inlet, $A$ is the cross-sectional area, and $F$ is a flux function, which is discontinuous at the inlet $(x=0)$ and at the two outlets. Section 3 treats dynamic solutions. All steady-state solutions of the problem are presented and classified in section 4.2. Examples, a control strategy for the optimal steady-state operation, and a discussion on the design of a settler can be found in section 4.3. To support the analytical results, a numerical algorithm and a few simulations are presented in section 5 . Conclusions can be found in section 6 .

Main results. The aim of the paper is to generalize the results in the preceding paper [11] to the case of nonconstant cross-sectional area and to give a control strategy for the steady-state behavior. One reason for the work was to answer some of the open questions addressed by Chancelier, de Lara, and Pacard [7]. Theorem 3.1 contains results on local existence and uniqueness of dynamic solutions. Theorems 4.4 and 4.6 contain the classifications of the steady-state solutions for a settler with strictly decreasing and constant cross-sectional area, respectively. Theorem 4.7 contains explicit formulas for the static control of the process. The numerical algorithm in section 5 is one outcome of this paper that has practical applications. 
The differences in method and results from the presentation of steady-state solutions by Chancelier, de Lara, and Parcard [7] are the following. Their approach starts by smoothing the point source and the discontinuity of the flux function at the feed inlet so that the well-known entropy condition and jump condition for scalar conservation laws with a continuous flux function can be used. In section 4 of the present paper, the steady-state solutions, including the effluent and the underflow concentrations, are obtained in a more direct way by using results from [11] involving condition $\Gamma$. With a slightly stronger constitutive assumption, the results of Chancelier, de Lara, and Pacard [7] are extended by a thorough description of the solutions in the thickening zone. In particular, it is shown that there is at most one discontinuity, the sludge blanket, in the thickening zone when the cross-sectional area is decreasing. Furthermore, it turns out that the steady-state behavior of a settler with constant cross-sectional area $A$ is a degenerate subcase of the case with a strictly decreasing $A$. For example, if a sludge blanket is possible, its level is uniquely determined by the feed concentration and the volume flows if $A$ is strictly decreasing, whereas it can be located anywhere if $A$ is constant. We also want to emphasize that the effluent and the underflow concentrations are generally not the same as the concentrations at the top and the bottom within the settler; see Lemma 4.1. For example, at the top of the clarification zone it is possible to have a specific high concentration of solids, such that the gravity settling downward is balanced by the volume flow upwards. Hence, the solids stay fixed, yielding a high concentration at the top and still the effluent concentration is zero. Analogously, the underflow concentration is generally larger than the bottom concentration in the thickening zone if the cross-sectional area is discontinuous between the bottom and the outflow pipe.

Related works. Away from the discontinuities of $F(u, \cdot)$ and the source, (1.1) can be written in the form $A(x) u_{t}+(A(x) f(u, A(x)))_{x}=0$, or

$$
u_{t}+f(u, A(x))_{x}=A^{\prime}(x) g(u, A(x)) .
$$

Equation (1.2) can be augmented to a nonstrictly hyperbolic system by adding the equation $a_{t}=0$, where $a=A(x)$. This type of inhomogeneous conservation law (with $f(\cdot, A)$ convex and $a=A(x)$ continuous) has been analyzed by, for example, Liu [31] and Isaacson and Temple [23], [24] with respect to the structure of elementary waves in a neighborhood of a state where a wave speed of (1.2) is zero (resonance) and the multiple steady states which then appear. In the present paper, we are interested in large discontinuities in a specific application where $f(\cdot, A)$ is nonconvex. Furthermore, the multiple steady states of (1.1) originate basically from the discontinuities of $F(u, \cdot)$ and the delta function in the source term. The latter can be included in $F$, and a discontinuity in $F(u, \cdot)$, say at $x=0$, can be replaced by a variable $a$ by adding the scalar equation $a_{t}+k(a)_{x}=0$ having Heaviside's step function $H(x)$ as the solution. For physical reasons (viscosity arguments), the function $k$ should not be chosen as the zero function; see [12]. Since also $a$ is discontinuous, (1.1) cannot easily be covered by the theory in [23], [24]. This is also indicated by the viscous profile analysis in [12], [15], where it is shown that the smoothing of a discontinuity in $F(u, \cdot)$ (to obtain a continuous $a$ ) should not be made without introducing a certain amount of viscosity in order to obtain physical stable solutions.

\section{Continuous sedimentation.}

2.1. The clarifier-thickener unit. Continuous sedimentation of solid particles in a liquid takes place in a clarifier-thickener unit or settler; see Fig. 2.1. Let $u(x, t)$ 


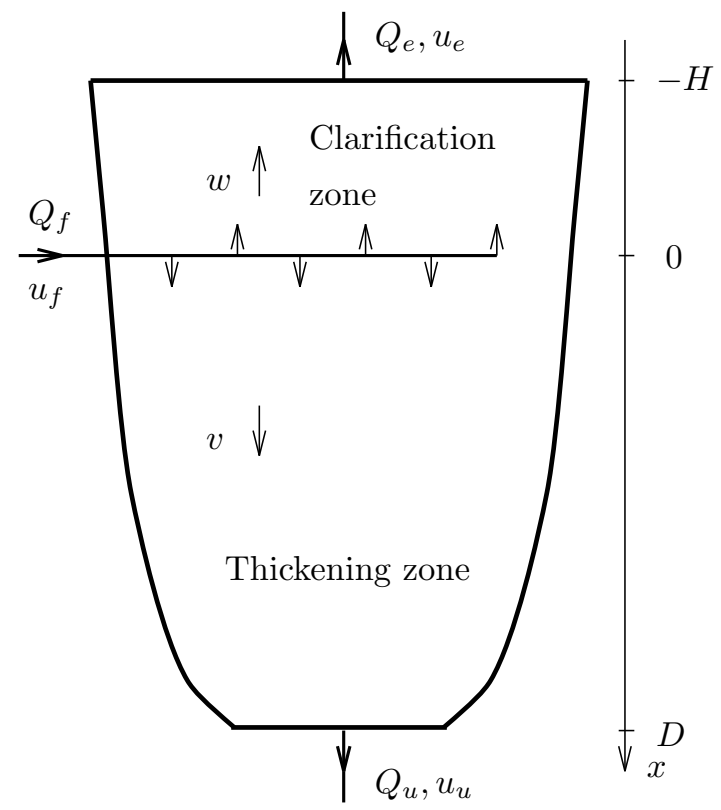

FIG. 2.1. Schematic picture of the continuous clarifier-thickener unit. The indices stand for: $e=$ effluent, $f=$ feed, and $u=$ underflow.

denote the concentration (mass per unit volume), where $t$ is the time coordinate and $x$ is the one-dimensional space coordinate; see Fig. 2.1. The height of the clarification zone is denoted by $H$ and the depth of the thickening zone by $D$. At $x=0$ the settler is fed with suspended solids at a concentration $u_{f}(t)$ and at a constant flow rate $Q_{f}$ (volume per unit time). A high concentration of solids is taken out at the underflow at $x=D$ at a flow rate $Q_{u}$. It is assumed that $0<Q_{u}<Q_{f}$. The effluent flow $Q_{e}$ at $x=-H$ is consequently defined by the flow condition $Q_{e}=Q_{f}-Q_{u}>0$. The cross-sectional area $A(x)$ is assumed to be $C^{1}$ for $-H<x<D$. Let us directly extend this function to the whole real axis by letting $A(x)=A(-H)$ for $x<-H$ and $A(x)=A(D)$ for $x>D$. We define the bulk velocities in the thickening and clarification zone as

$$
v(x)=\frac{Q_{u}}{A(x)}, \quad w(x)=\frac{Q_{e}}{A(x)},
$$

with directions shown in Fig. 2.1. For the source term, it will be convenient to use the notation

$$
S(t)=Q_{f} u_{f}(t), \quad s(t)=\frac{S(t)}{A(0)},
$$

where $S(t)$ is the mass per unit time entering the settler. The mass per unit time leaving the settler through the outlets is the sum of $Q_{e} u_{e}(t)$ and $Q_{u} u_{u}(t)$, where the effluent concentration $u_{e}(t)$ and the underflow concentration $u_{u}(t)$ should be determined by the model.

The volume flows $Q_{f}, Q_{u}$, and, hence, $Q_{e}$ may vary with time. The generalization to the case when $Q_{f}(t)$, etc. are piecewise smooth is straightforward, and to avoid cumbersome notation we assume that the $Q$-flows are constant. 
2.2. A constitutive assumption. Denote the maximum packing concentration of solid particles or sludge by $u_{\max }$. In batch sedimentation there is no bulk flow and the solids settle due to gravity. The settling velocity is assumed to depend only on the concentration of particles, $v_{\text {settl }}(u)$. This assumption was introduced by Kynch [28]. The downward flux of sludge (mass per unit time and unit area), the batch settling flux, is defined as $\phi(u)=u v_{\text {settl }}(u)$. We shall use a common batch settling flux $\phi$ with the following properties; see Fig. 2.2,

$$
\begin{aligned}
& \phi \in C^{2}, \\
& \phi(0)=\phi\left(u_{\max }\right)=0, \\
& \phi(u)>0, \quad u \in\left(0, u_{\max }\right), \\
& \phi \text { has exactly one inflection point } u_{\text {infl }} \in\left(0, u_{\max }\right), \\
& \phi^{\prime \prime}(u)<0, \quad u \in\left[0, u_{\text {inf }}\right) .
\end{aligned}
$$

Chancelier, de Lara, and Pacard [7] use the weaker condition $v_{\text {settl }}^{\prime}(u)<0$ for $u \geq 0$, which admits more than one inflection point of $\phi$. (Note that $v_{\text {settl }}(u)=\phi(u) / u$ implies $v_{\text {settl }}^{\prime}(u)=\psi(u) / u^{2}$ with $\psi(u)=\phi^{\prime}(u) u-\phi(u)$. If $\phi$ satisfies $(2.2)$, then $\psi(0)=0, \psi\left(u_{\max }\right)=\phi^{\prime}\left(u_{\max }\right) u_{\max } \leq 0$ and $\psi^{\prime}(u)=\phi^{\prime \prime}(u) u$. Hence, $\psi(u)<0$ for $u \in\left(0, u_{\max }\right)$ and $v_{\text {settl }}^{\prime}(u)<0$ for $u \in\left(0, u_{\max }\right)$.) With our choice of $\phi$, it is possible to obtain a detailed description of the steady-state solutions in the thickening zone; see section 4.2. Furthermore, by letting $u_{\max }$ be finite (instead of infinite as in [7]) with $\phi^{\prime}\left(u_{\max }\right)<0$, there are more qualitatively different cases (see section 4 ) that might be of interest in chemical engineering; cf. [1], [8].

2.3. A mathematical model. In continuous sedimentation the volume flows $Q_{u}$ and $Q_{e}$ give rise to the flux terms $v(x) u$ and $-w(x) u$, respectively, which are superimposed on the batch settling flux $\phi(u)$ to yield the total flux in the clarification and the thickening zones. We extend the space variable to the whole real line by assuming that outside the settler the particles have the same speed as the liquid. Thus, we define a total flux function, built up by the flux functions in the respective region, as

$$
F(u, x)= \begin{cases}g_{e}(u)=-w(-H) u, & x<-H, \\ g(u, x)=\phi(u)-w(x) u, & -H<x<0, \\ f(u, x)=\phi(u)+v(x) u, & 0<x<D, \\ f_{u}(u)=v(D) u, & x>D .\end{cases}
$$

Typical flux curves $\phi, f$, and $g$ are shown in Fig. 2.2. In the following, we write $g(u,-H)$ for the limits $g(u,-H+0)$, etc.

Assume that the $Q$-flows and, hence, the flux function $F$ given by (2.3) are known as well as the feed concentration $u_{f}$ (hence the source function $S$ ). The concentration distribution $u(x, t)$ in the settler and the two functions $u_{e}$ and $u_{u}$ are unknown. Introduce the limits

$$
u_{ \pm}(t)=\lim _{\delta \searrow 0} u( \pm \delta, t)
$$




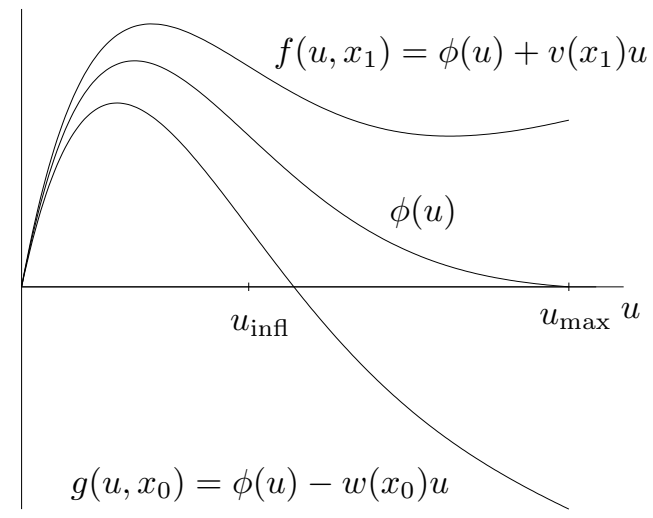

Fig. 2.2. The flux curves $\phi, f\left(\cdot, x_{1}\right)$ and $g\left(\cdot, x_{0}\right)$, where $-H<x_{0}<0<x_{1}<D$.

The conservation law, preservation of mass, can be used to obtain, for $t>0$,

$$
\begin{array}{ll}
\partial_{t} u+\partial_{x} g_{e}(u)=0, & x<-H, \\
A(x) \partial_{t} u+\partial_{x}(A(x) g(u, x))=0, & -H<x<0, \\
A(x) \partial_{t} u+\partial_{x}(A(x) f(u, x))=0, & 0<x<D, \\
\partial_{t} u+\partial_{x} f_{u}(u)=0, & x>D, \\
g(u(-H+0, t),-H)=g_{e}\left(u_{e}(t)\right), & \\
f\left(u_{+}(t), 0\right)=g\left(u_{-}(t), 0\right)+s(t), & \\
f_{u}\left(u_{u}(t)\right)=f(u(D-0, t), D), & x \in \mathbb{R} . \\
u(x, 0)=u_{0}(x), &
\end{array}
$$

We assume that $u_{0}(x), u_{f}(t) \in\left[0, u_{\max }\right]$. Note that the speed of the characteristics in the region $x<-H$ is $-w(-H)<0$ and in the region $x>D$ is $v(D)>0$. This means that the solution is known if $u(x, t), u_{e}(t) \equiv u(-H-0, t)$, and $u_{u}(t) \equiv u(D+0, t)$ are known for $-H<x<D$ and $t>0$. The weak formulation of (2.4) is

$$
\begin{aligned}
\int_{0}^{\infty} \int_{-\infty}^{\infty} A(x)\left(u \partial_{t} \varphi+F(u, x) \partial_{x} \varphi\right) & d x d t+\int_{-\infty}^{\infty} A(x) u_{0}(x) \varphi(x, 0) d x \\
& +\int_{0}^{\infty} S(t) \varphi(0, t) d t=0, \quad \varphi \in C_{0}^{\infty}\left(\mathbb{R}^{2}\right),
\end{aligned}
$$

with $F$ given by (2.3). By standard arguments it can be shown that (2.4) is equivalent to $(2.5)$ if $u(x, t)$ is a function that is smooth except along $x=-H, x=0$, and $x=D$. A function $u(x, t)$ is said to be piecewise smooth if it is bounded and $C^{1}$ except along a finite number of $C^{1}$-curves such that the left and right limits of $u$ along discontinuity curves exist. A function of one variable is said to be piecewise monotone if there are at most a finite number of points where a shift of monotonicity occurs.

3. Results on dynamic solutions. In [11], existence and uniqueness results for (2.4) were given in the case of a constant cross-sectional area $A$. The construction of solutions in that case can be generalized rather straightforward to the case of varying $A(x)$. It depends heavily on a generalized entropy condition, condition $\Gamma$, handling the solution at the discontinuities of $F(u, \cdot)$, and the notion of a regular Cauchy 
problem. Since these concepts need cumbersome notation, and since they have been described thoroughly in [10]-[12], we refer to those papers for the definitions and examples. Briefly described, condition $\Gamma$ converts flow conditions (conservation of mass) into well-defined boundary values on both sides of a discontinuity of $F(u, \cdot)$. The regularity assumption is made only for technical reasons and causes no restriction in the application to sedimentation. Here we shall formulate the theorem, but only outline the proof.

THEOREM 3.1. Assume that $A(x), u_{0}(x)$, and $u_{f}(t)$ are piecewise monotone, $u_{0}(x)$ and $u_{f}(t)$ are piecewise smooth, $A(x) \in C^{1}(-H, D), u_{f}(t)$ has bounded derivative, and $0 \leq u_{0}(x), u_{f}(t) \leq u_{\max }, x \in \mathbb{R}, t \geq 0$. If (2.4) is regular, then there exists a unique piecewise smooth function $u(x, t), x \in \mathbb{R}, t \in[0, \varepsilon)$ for some $\varepsilon>0$, satisfying condition $\Gamma$, and with $u_{ \pm}(t), u_{e}(t)$, and $u_{u}(t)$ piecewise monotone. This solution satisfies $0 \leq u(x, t), u_{e}(t), u_{u}(t) \leq u_{\max }$ for $x \in \mathbb{R}, t \in[0, \varepsilon)$.

Proof. The construction of solutions consists in finding boundary functions on either side of the discontinuities of $F(u, \cdot)$ such that the method of characteristics can be applied, for small $t>0$, to the initial boundary value problem that arises. Away from the discontinuities of $F(u, \cdot)$, the solution is determined by the characteristics from the $x$-axis. In the thickening zone, for example, the equation is $A(x) \partial_{t} u+$ $\partial_{x}(A(x) f(u, x))=0$ and it can be written

$$
\partial_{t} u+\partial_{u} f(u, x) u_{x}=-\frac{A^{\prime}(x)}{A(x)} \phi(u) .
$$

Hence, a characteristic $x=x(t)$ and its concentration values are governed by the equations

$$
\begin{aligned}
& \frac{d x}{d t}=\partial_{u} f(u, x), \\
& \frac{d u}{d t}=-\frac{A^{\prime}(x)}{A(x)} \phi(u) .
\end{aligned}
$$

Now consider the discontinuity of $F(u, \cdot)$ at $x=0$. It is straightforward to check that the boundary functions, used in the proof in [11], on either side of the $t$-axis will depend on the functions $f(\cdot, 0), g(\cdot, 0), S$, and on functions of the type $\tilde{u}(0+, t)$, where $\tilde{u}$ is the unique solution (Kružkov [27]) of the auxiliary problem

$$
\begin{aligned}
& A(x) \partial_{t} \tilde{u}+\partial_{x}(A(x) f(\tilde{u}, x))=0, \\
& \tilde{u}(x, 0)= \begin{cases}a, & x<0, \\
u(x, 0), & x>0,\end{cases}
\end{aligned}
$$

where $a$ is a constant, depending on $A(0)$. The technical assumptions on regularity concern piecewise smoothness and piecewise monotonicity of $\tilde{u}(0+, \cdot)$ and the corresponding function to left of the $t$-axis. These two functions are used in formulas depending on $A(0)$ that finally define the correct boundary functions; see [11].

The proof of uniqueness of the constructed solution consists in treating several cases. The division of these depends on the continuity and monotonicity both of the functions $\tilde{u}(0+, t), f(\tilde{u}(0+, t), 0)$, etc. for small $t>0$ and of $u(x, 0)$ for $x$ in a neighborhood of $x=0$. Arguments such as " $\partial_{u} f(\tilde{u}(0+, t), 0)<0$ for small $t>0$ implies that $\tilde{u}(0+, t)$ is uniquely determined by the characteristics from the positive $x$-axis" still hold by continuity of $A$ and $A^{\prime}$ and by equations (3.1). It is also of 
importance that the jump and entropy conditions for a discontinuity along the $t$ axis of the solution of (3.2) are independent of $A(x)$. The jump condition is simply $f\left(u_{-}, 0\right)=f\left(u_{+}, 0\right)$, and the entropy condition reads $\frac{f(\tilde{u}, 0)-f\left(u_{-}, 0\right)}{\tilde{u}-u_{-}} \geq 0$ for all $\tilde{u}$ between $u_{-}$and $u_{+}$.

Finally, the boundedness condition on the solution is proved as follows. With $U=A(x) u$, the equation in the thickening zone is $\partial_{t} U+\partial_{x}(A(x) f(U / A(x), x))=0$ and the ordering principle for two solutions $U$ and $U_{1}$ holds (Kružkov [27]): $0 \leq$ $U(x, 0) \leq U_{1}(x, 0)$ implies $0 \leq U(x, t) \leq U_{1}(x, t)$. Now $U_{1}(x, t) \equiv A(x) u_{\max }$ is a solution, because $\phi\left(u_{\max }\right)=0$ implies

$$
\partial_{t} U_{1}+\partial_{x}\left(A(x) f\left(U_{1} / A(x), x\right)\right)=0+\partial_{x}\left(A(x) \phi\left(u_{\max }\right)+Q_{u} u_{\max }\right)=0 .
$$

For the clarification zone, replace $Q_{u}$ by $-Q_{e}$ and $f$ by $g$. It follows that $0 \leq u \leq u_{\max }$ for the concentrations $u$ carried by the characteristics from the $x$-axis. The same bound can be obtained for the boundary functions at the discontinuities of $F(u, \cdot)$ (see [11]) by using the cross-sectional areas $A(-H), A(0)$, and $A(D)$ at the respective discontinuity.

4. Steady-state behavior. In order to capture the steady-state behavior of the settler for different values of $u_{f}$ and the $Q$-flows, a number of characteristic concentrations and fluxes are defined in section 4.1. One of these is the limiting flux, introduced by Chancelier, de Lara, and Pacard [7], which determines whether there is an overflow or not, as well as the type of solution in the clarification zone. It turns out that when $A^{\prime}(x)<0$ in the thickening zone, there is actually only one possibility for a stationary discontinuity. This is usually referred to as the sludge blanket. We shall use this definition, whereas Chancelier, de Lara, and Pacard [7] define the sludge blanket as being the uppermost discontinuity between clear water and solids. This appears in the clarification zone or at the feed level. The following terms are often used for the steady-state behavior. The settler is said to be

- in optimal operation if there is a sludge blanket in the thickening zone and the concentration in the clarification zone is zero;

- underloaded if no sludge blanket is possible and the concentration in the clarification zone is zero;

- overloaded if the effluent concentration $u_{e}>0$.

As we shall see below, there are steady-state solutions which do not fit into any of these three definitions. For example, there may be a discontinuity in the clarification zone but the effluent concentration is still zero.

Owing to the appearance of the sludge blanket, we introduce the sludge blanket flux $\Phi_{\mathrm{sb}}\left(x_{1}\right)$, which is a decreasing function of the sludge blanket depth $x_{1}$. There are roughly three different types of stationary solution in the thickening zone. If the applied flux in the thickening zone lies in the range of $\Phi_{\mathrm{sb}}$, then there will be a sludge blanket (possibly a degenerate discontinuity); see Fig. 4.2. If the applied flux is lower (higher), then the solution is continuous and low (high), respectively.

Section 4.3 contains some interpretations of the results obtained in section 4.2 with emphasis on the static control of the sludge blanket depth by using $Q_{u}$ as a control parameter.

4.1. Definitions and notation. First, we define some characteristic concentrations that depend on the flux functions $f$ and $g$. For fixed $x \in(-H, 0)$, denote the unique strictly positive zero of $g(\cdot, x)$ by $u_{z}(x)$, so that

$$
u_{z}(x)>0, \quad\left(u_{z}(x), x\right)=0 ;
$$


see Fig. 4.1. Write $u_{z}(-H)$ instead of $u_{z}(-H+0)$. For very high bulk velocities $w(x)$ such that $g(\cdot, x)$ is decreasing, we define $u_{z}(x)=0$. If this happens, some of the cases in this paper will be empty and we shall refrain from commenting upon this anymore. The concentration $u_{z}(x)$ is such that the gravity settling downward is balanced by the volume flow upward. Hence, a layer of sludge in the clarification zone with this concentration will be at rest.

Let $h(u, v)=\phi(u)+v u$, where $\phi$ has properties (2.2). Then $f(u, x)=h(u, v(x))$. Note that the inflection point $u_{\text {inf }}$ of $\phi$ is the same as the inflection point of $h(\cdot, v)$ independently of $v$. It turns out that the strict local minimizer of $h(\cdot, v)$ in the interval $\left(0, u_{\max }\right)$, denoted $\check{u}(v)$, is important for the behavior of the solution in the thickening zone. It is defined implicitly by

$$
\partial_{u} h(\check{u}(v), v)=\phi^{\prime}(\check{u}(v))+v=0
$$

as long as $\phi^{\prime \prime}(\check{u}(v)) \neq 0$. The properties $(2.2)$ of $\phi$ imply that $u_{\text {inf }}<\check{u}(v)<u_{\max }$ and that for such values of $v$

$$
\check{u}^{\prime}(v)=-\frac{1}{\phi^{\prime \prime}(\check{u}(v))}<0 .
$$

Therefore, we define

$$
\bar{v}=-\phi^{\prime}\left(u_{\max }\right)>0 \quad \Longleftrightarrow \quad \partial_{u} h\left(u_{\max }, \bar{v}\right)=0,
$$

which is the bulk velocity such that the minimizer $\check{u}(v)$ equals $u_{\max }$, and

$$
\overline{\bar{v}}=\inf \{v: h(\cdot, v) \text { is strictly increasing }\} .
$$

Hence, $\breve{u}(v)$ decreases from $u_{\max }$ to $u_{\text {infl }}$ as $v$ increases from $\bar{v}$ to $\overline{\bar{v}}$. Define, for fixed $x \in(0, D)$,

$$
\begin{aligned}
& u_{M}(x)= \begin{cases}u_{\max }, & v(x) \leq \bar{v}, \\
\check{u}(v(x)), & \bar{v}<v(x)<\overline{\bar{v}}, \\
u_{\text {infl }}, & v(x) \geq \overline{\bar{v}}\end{cases} \\
& u_{m}(x)=\min \left\{u: f(u, x)=f\left(u_{M}(x), x\right)\right\} ;
\end{aligned}
$$

see Fig. 4.1. Note that the assumption $A^{\prime}(x)<0$ in the thickening zone implies that

$$
\begin{array}{ll}
v^{\prime}(x)>0, & 0<x<D, \\
u_{M}^{\prime}(x)<0, & \bar{v}<v(x)<\overline{\bar{v}}, \\
u_{m}^{\prime}(x)>0, & 0<v(x)<\overline{\bar{v}},
\end{array}
$$

and that all these derivatives are continuous.

A term frequently used to describe the behavior of the settler is the limiting flux, which denotes the maximum flux capacity of the underflow. Chancelier, de Lara, and Pacard [7] introduce the following definition, which we apply directly to our flux function $f(\cdot, 0)$. Given $Q_{u}$ and $u_{f}$, define the limiting flux as

$$
\begin{aligned}
\Phi_{\lim }=A(0) \min _{u_{f} \leq u \leq u_{\max }} f(u, 0) \\
= \begin{cases}A(0) f\left(u_{f}, 0\right), & u_{f} \in\left[0, u_{m}(0)\right] \cup\left[u_{M}(0), u_{\max }\right], \\
A(0) f\left(u_{M}(0), 0\right), & u_{f} \in\left(u_{m}(0), u_{M}(0)\right)\end{cases}
\end{aligned}
$$




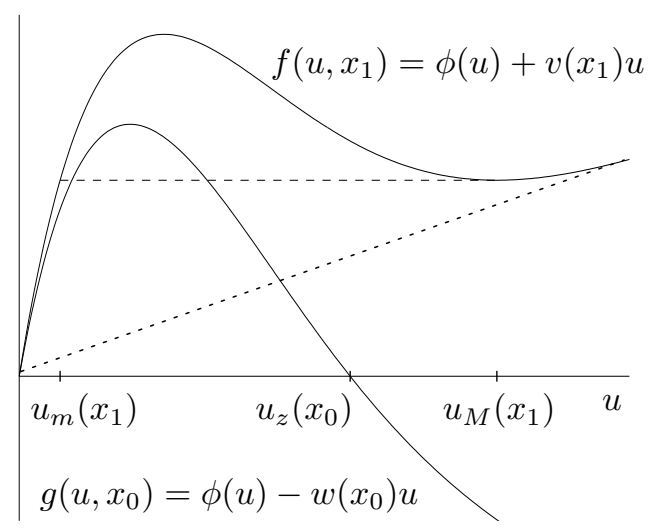

FIG. 4.1. The zero $u_{z}\left(x_{0}\right)$ of $g\left(\cdot, x_{0}\right)$ and the two characteristic concentrations of $f\left(\cdot, x_{1}\right)$ in the case when $\bar{v}<v\left(x_{1}\right)<\overline{\bar{v}}$. The slope of the dotted line is $v\left(x_{1}\right)$ and $-H<x_{0}<0<x_{1}<D$.

Note that $\Phi_{\text {lim }}$ is independent of $Q_{f}$ and $Q_{e}$ and that $\Phi_{\text {lim }}$ is a continuous increasing function of $u_{f}$, constant on the interval $\left(u_{m}(0), u_{M}(0)\right)$, strictly increasing otherwise.

Let $u(x, t) \equiv u_{s}(x)$ denote a steady-state, or stationary, solution of (2.4) with

$$
u_{s}(x)=\left\{\begin{array}{lr}
u_{l}(x), & -H<x<0, \\
u_{r}(x), & 0<x<D .
\end{array}\right.
$$

Hence, $u_{-}=u_{l}(0-), u_{+}=u_{r}(0+)$, and we let $u_{l}(-H) \equiv u_{l}(-H+0)$ and $u_{r}(D) \equiv$ $u_{r}(D-0)$. Denote the steady-state fluxes in the clarification and the thickening zone by $\Phi_{\text {clar }} \geq 0$ and $\Phi_{\text {thick }} \geq 0$, respectively, so that $S=\Phi_{\text {clar }}+\Phi_{\text {thick }}$. (Recall that $S=Q_{f} u_{f}$.) Then $u_{l}(x)$ and $u_{r}(x)$ are defined implicitly by the equations

$$
\begin{array}{cr}
\Phi_{\text {clar }}=-A(x) g\left(u_{l}(x), x\right), & -H<x<0, \\
\Phi_{\text {thick }}=A(x) f\left(u_{r}(x), x\right), & 0<x<D,
\end{array}
$$

and the effluent and underflow concentrations satisfy

$$
\begin{aligned}
\Phi_{\text {clar }} & =Q_{e} u_{e}, \\
\Phi_{\text {thick }} & =Q_{u} u_{u} .
\end{aligned}
$$

In section 4.2 , it turns out that, when $A^{\prime}(x)<0$ in the thickening zone, there is actually only one possibility for a stationary discontinuity, the sludge blanket. If $x \in(0, D)$ is the location of the discontinuity, then the left and right limits of the discontinuity are $u_{m}(x)$ and $u_{M}(x)$; see Fig. 4.1. To describe this situation we define the function

$$
\Phi_{\mathrm{sb}}(x)=A(x) f\left(u_{M}(x), x\right)= \begin{cases}Q_{u} u_{\max }, & v(x) \leq \bar{v}, \\ A(x) \phi\left(u_{M}(x)\right)+Q_{u} u_{M}(x), & \bar{v}<v(x)<\overline{\bar{v}} \\ A(x) \phi\left(u_{\mathrm{infl}}\right)+Q_{u} u_{\mathrm{infl}}, & v(x) \geq \overline{\bar{v}}\end{cases}
$$

When $x$ is the depth of the sludge blanket, this function gives the sludge blanket flux. Differentiating and using $\partial_{u} f\left(u_{M}(x), x\right) \equiv 0$ for $\bar{v}<v(x)<\overline{\bar{v}}$ gives

$$
\Phi_{\mathrm{sb}}^{\prime}(x)=A^{\prime}(x) \phi\left(u_{M}(x)\right)= \begin{cases}0, & v(x) \leq \bar{v} \\ A^{\prime}(x) \phi\left(u_{M}(x)\right), & \bar{v}<v(x)<\overline{\bar{v}} \\ A^{\prime}(x) \phi\left(u_{\mathrm{inf}}\right), & v(x) \geq \overline{\bar{v}}\end{cases}
$$


4.2. The steady-state solutions. A steady-state solution of (2.4) is obtained by determining the stationary concentration distribution $u_{s}(x)$ (in terms of $u_{l}(x)$ and $\left.u_{r}(x)\right)$ and the constant effluent and underflow concentrations $u_{e}$ and $u_{u}$. Supposing that $u_{s}(x)$ is piecewise smooth and piecewise monotone, Theorem 3.1 guarantees uniqueness. Furthermore, we assume that $A^{\prime}(x)<0$ in the thickening zone. Then the properties (2.2) of $\phi$ are sufficient to conclude that there is at most one discontinuity in the thickening zone and that $u_{r}(x)$ is increasing. The procedure for obtaining the steady-state solutions consists in extracting all possible combinations of the concentrations at the point source and at the two outlets from [11] and combining these with the steady-state solutions in the clarification and thickening zone. However, we shall only describe the main line here and refer to the appendix for the tedious details.

If $u_{f}=0$, then $0=S=\Phi_{\text {clar }}+\Phi_{\text {thick }}$ and since both these fluxes are nonnegative, they must be zero. Hence, $u_{s}(x) \equiv 0$ and $u_{e}=u_{u}=0$. We assume from now on that $u_{f}>0$.

LEMMA 4.1. Necessary conditions on the concentrations at the outlets at steady state are

- either $u_{l}(-H)=u_{e}=0$ or $u_{l}(-H) \geq u_{z}(-H)$ with $u_{e}=u_{l}(-H)-$ $\phi\left(u_{l}(-H)\right) / w(-H)$

- $u_{r}(D) \in\left[0, u_{m}(D)\right] \cup\left[u_{M}(D), u_{\max }\right]$ with $u_{u}=u_{r}(D)+\phi\left(u_{r}(D)\right) / v(D)$.

Proof. See section 9 in [11].

The lemma implies that the effluent and underflow concentrations satisfy $u_{e} \leq$ $u_{l}(-H)$ and $u_{u} \geq u_{r}(D)$ with equality if and only if the concentrations are zero or $u_{\max }$.

LEMma 4.2. Possible concentration distributions and fluxes in the clarification zone at steady state are

CI. $u_{l}(x)=0, x \in(-H, 0)$, with $\Phi_{\text {clar }}=0$;

CII. $u_{l}(x)=\left\{\begin{array}{lc}0, & -H<x<x_{0} \\ u_{z}(x), & x_{0}<x<0\end{array}\right.$ for some $x_{0} \in[-H, 0)$ with $\Phi_{\text {clar }}=0$ (here, $x_{0}=-H$ means $\left.u_{l}(x) \equiv u_{z}(x)\right)$;

CIII. $u_{l}(x)$ is smooth with $u_{l}(x)>u_{z}(x), x \in(-H, 0)$, with $\Phi_{\text {clar }}>0$.

Furthermore, when $u_{l}(x) \geq u_{z}(x)$, then

$$
u_{l}^{\prime}(x) \lessgtr 0 \quad \Longleftrightarrow \quad A^{\prime}(x) \lessgtr 0 .
$$

The steady-state solutions in the thickening zone are a bit more complicated to sort out. The appearance of a sludge blanket is particularly important. So far, we have associated the sludge blanket with a discontinuity. Before presenting Lemma 4.3 and Theorem 4.4, we augment the concept of the sludge blanket at $x_{1}$ by including the case when $Q_{u}$ is so large or $A(x)$ so small that $f\left(\cdot, x_{1}\right)$ is increasing, i.e., when $v\left(x_{1}\right) \geq \overline{\bar{v}}$. Then the discontinuity degenerates, since $u_{m}\left(x_{1}\right)=u_{M}\left(x_{1}\right)=u_{\text {infl }}$, by (4.1) (TIIIB in Lemma 4.3); see the rightmost graph of Fig. 4.3.

The assumption $A^{\prime}(x)<0$ for $0<x<D$ implies that $v^{\prime}(x)>0$ and, by (4.2), that

$$
\Phi_{\mathrm{sb}}^{\prime}(x) \begin{cases}=0, & v(x) \leq \bar{v} \\ <0, & v(x)>\bar{v} .\end{cases}
$$

Hence, $\Phi_{\mathrm{sb}}(0) \geq \Phi_{\mathrm{sb}}(D)$ with equality if and only if $v(D) \leq \bar{v}$.

Lemma 4.3. Assume that $A^{\prime}(x)<0$ for $0<x<D$. Then there are three different possible types of concentration distribution in the thickening zone at steady state. In 
all cases, $u_{r}$ is smooth with $u_{r}^{\prime}(x)>0$ when $u_{r}(x) \in\left(0, u_{\max }\right)$ except possibly at the sludge blanket. The types are the following:

TI. $u_{r}(x)<u_{m}(x), x \in(0, D)$, with $\Phi_{\text {thick }} \leq \Phi_{\mathrm{sb}}(D)$.

TII. A. $u_{r}(x)=u_{\max }, x \in(0, D)$, with $\Phi_{\text {thick }} \geq \Phi_{\mathrm{sb}}(0)$.

B. $u_{M}(x)<u_{r}(x)<u_{\max }, x \in(0, D)$, with $v(0)>\bar{v}$ and $\Phi_{\text {thick }} \geq \Phi_{\mathrm{sb}}(0)$.

TIII. There exists a sludge blanket at $x_{1} \in(0, D)$, which is uniquely determined by $\Phi_{\mathrm{sb}}(D)<\Phi_{\text {thick }}=\Phi_{\mathrm{sb}}\left(x_{1}\right)<\Phi_{\mathrm{sb}}(0)$ (for given $\left.\Phi_{\text {thick }}\right)$. Also $\bar{v}<v\left(x_{1}\right)$ holds. The solution satisfies

$$
0<u_{r}(x) \begin{cases}<u_{m}(x), & 0<x<x_{1}, \\ >u_{M}(x), & x_{1}<x<D,\end{cases}
$$

with $u_{r}\left(x_{1}-0\right)=u_{m}\left(x_{1}\right), u_{r}\left(x_{1}+0\right)=u_{M}\left(x_{1}\right), u_{r}(x)<u_{\max }$ for $x \in(0, D)$, and either

A. $v\left(x_{1}\right)<\overline{\bar{v}}: u_{r}(x)$ is discontinuous only at $x_{1}$ with $u_{r}^{\prime}(x) \rightarrow \infty$ as $x \searrow x_{1}$; cf. Fig. 4.2; or

B. $v\left(x_{1}\right) \geq \overline{\bar{v}}: u_{r}(x)$ is continuous and $u_{m}\left(x_{1}\right)=u_{M}\left(x_{1}\right)=u_{\text {infl }}$; cf. the rightmost graph in Fig. 4.3.

Now we shall put together the stationary solutions $u_{l}(x)$ and $u_{r}(x)$ obtained in Lemmas 4.2 and 4.3 by using Lemma A.1 of the appendix.

THEOREM 4.4. Referring to the different types of solution, CI, etc., in Lemmas 4.2 and 4.3, the following classification of steady-state behavior holds for a settler with $A^{\prime}(x)<0$ for $0<x<D$. The symbol $\emptyset$ denotes an impossible case.

$\star S<\Phi_{\text {lim }}$. The solution in the clarification zone is of type CI with $u_{e}=0$ and $\Phi_{\text {clar }}=0$. Hence $\Phi_{\text {thick }}=S$ and $u_{u}=S / Q_{u}$. In the thickening zone the solutions are the following when $v(D)>\bar{v} \Leftrightarrow \Phi_{\mathrm{sb}}(D)<\Phi_{\mathrm{sb}}(0)$ :

\begin{tabular}{|c||c|c|c|}
\hline & & $\Phi_{\mathrm{sb}}(D)$ & \\
& $S \leq \Phi_{\mathrm{sb}}(D)$ & $<S<\Phi_{\mathrm{sb}}(0)$ & $S \geq \Phi_{\mathrm{sb}}(0)$ \\
\hline \hline $0<u_{f} \leq u_{M}(0)$ & & & $\emptyset$ \\
\cline { 1 - 1 } $\begin{array}{c}u_{M}(0) \\
<u_{f} \leq u_{\max }\end{array}$ & $<\operatorname{TI}, u_{+}$ & TIII, $u_{+}$ & TIIB, \\
& $<\min \left(u_{f}, u_{m}(0)\right)$ & $<\min \left(u_{f}, u_{m}(0)\right)$ & $u_{M}(0) \leq u_{+}<u_{f}$ \\
\hline
\end{tabular}

For $v(D) \leq \bar{v} \Leftrightarrow \Phi_{\mathrm{sb}}(x) \equiv \Phi_{\mathrm{sb}}(0)$ the following holds:

\begin{tabular}{|c||c|c|}
\hline & $S<\Phi_{\mathrm{sb}}(0)$ & $S \geq \Phi_{\mathrm{sb}}(0)$ \\
\hline \hline $0<u_{f} \leq u_{\max }$ & TI, $u_{+}<\min \left(u_{f}, u_{m}(0)\right)$ & $\emptyset$ \\
\hline
\end{tabular}

$S=\Phi_{\lim }$. CI or CII $\left(u_{-}=0\right.$ or $\left.u_{-}=u_{z}(0)\right)$ with $u_{e}=0$ and $\Phi_{\text {clar }}=0$. Hence, $\overline{\Phi_{\text {thick }}}=S$ and $u_{u}=S / Q_{u}$. For $v(D)>\bar{v}$ the following holds:

\begin{tabular}{|c||c|c|c|c|}
\hline & $S \leq \Phi_{\mathrm{sb}}(D)$ & $\begin{array}{c}\Phi_{\mathrm{sb}}(D) \\
<S<\Phi_{\mathrm{sb}}(0)\end{array}$ & $S=\Phi_{\mathrm{sb}}(0)$ & $S>\Phi_{\mathrm{sb}}(0)$ \\
\hline \hline $\begin{array}{c}0<u_{f} \\
<u_{m}(0)\end{array}$ & $\begin{array}{c}\text { TI, } u_{+} \\
=u_{f}=u_{z}(0)\end{array}$ & $\begin{array}{c}\text { TIII, } u_{+} \\
=u_{f}=u_{z}(0)\end{array}$ & $\emptyset$ & \multirow{2}{*}{$\emptyset$} \\
\cline { 1 - 3 } $\begin{array}{c}u_{m}(0) \leq u_{f} \\
\leq u_{M}(0)\end{array}$ & $\emptyset$ & $\emptyset$ & $\begin{array}{c}\text { TIIA }(v(0) \leq \bar{v}) \\
\text { or } \mathrm{B}, u_{f} \leq u_{z}(0) \\
u_{M}(0)=u_{+}\end{array}$ & \\
\cline { 1 - 3 } $\begin{array}{c}u_{M}(0) \\
<u_{f} \leq u_{\max }\end{array}$ & & $\emptyset$ & $\emptyset$ & $\begin{array}{c}\text { TII }, u_{+} \\
=u_{f}=u_{z}(0)\end{array}$ \\
\hline
\end{tabular}


For $v(D) \leq \bar{v}$ the following holds:

\begin{tabular}{|l||c|c|c|}
\hline & $S<\Phi_{\mathrm{sb}}(0)$ & $S=\Phi_{\mathrm{sb}}(0)$ & $S>\Phi_{\mathrm{sb}}(0)$ \\
\hline \hline \multirow{2}{*}{$0<u_{f}<u_{m}(0)$} & TI, & $\emptyset$ & \\
\cline { 1 - 2 } & $u_{+}=u_{f}=u_{z}(0)$ & TIIA, $u_{f} \leq u_{z}(0)$ & $\emptyset$ \\
$u_{m}(0) \leq u_{f} \leq u_{\max }$ & $\emptyset$ & $\leq u_{M}(0)=u_{+}$ & \\
\hline
\end{tabular}

$\star \underline{S>\Phi_{\text {lim }}}$. CIII with $u_{-}>u_{z}(0), \Phi_{\text {thick }}=\Phi_{\text {lim }}, \Phi_{\text {clar }}=S-\Phi_{\text {lim }}, u_{e}=$ $\Phi_{\text {clar }} / \overline{Q_{e}>0, u_{u}}=\Phi_{\lim } / Q_{u}$. Then

\begin{tabular}{|l||c|c|}
\hline & $\Phi_{\lim }<\Phi_{\mathrm{sb}}(0)$ & $\Phi_{\lim } \geq \Phi_{\mathrm{sb}}(0)$ \\
\hline \hline $0<u_{f}<u_{m}(0)$ & TI, $u_{-}=u_{+}=u_{f}$ & $\emptyset$ \\
\hline & $\emptyset$ & TIIA $(v(0) \leq \bar{v})$ or $\mathrm{B}$, \\
$u_{m}(0) \leq u_{f} \leq u_{M}(0)$ & $\emptyset$ & $u_{f}<u_{-}<u_{M}(0)=u_{+}$ \\
\hline & $\emptyset$ & TIIA $(v(0) \leq \bar{v})$ or $\mathrm{B}$, \\
$u_{M}(0)<u_{f} \leq u_{\max }$ & $\emptyset$ & $u_{-}=u_{+}=u_{f}$ \\
\hline
\end{tabular}

The tables and the equation $f\left(u_{+}, 0\right)=g\left(u_{-}, 0\right)+s$ determine the concentrations $u_{-} \leq u_{+}$uniquely.

For a discussion on the different cases above we refer to section 4.3.

Corollary 4.5. Assume that $A^{\prime}(x)<0$ for $x \in(0, D)$. Given $Q_{f}, Q_{u}$, and $u_{f}$, there is precisely one steady-state solution of (2.4) except for the clarification zone when $S=\Phi_{\text {lim }}$, corresponding to the solution-type CII of Lemma 4.2.

Although the steady-state solutions in the case of a constant cross-sectional area have been presented in [11], we shall here give a classification similar to that in Theorem 4.4. When $A$ is constant, $v, u_{m}, u_{M}, u_{z}$, and $\Phi_{\mathrm{sb}}$ are constants and $u_{s}(x)$ is piecewise constant. Lemma 4.2 gives the possibilities for $u_{l}(x)$. It is appropriate to redefine the types of solution in the thickening zone slightly so that the sludge blanket in type TIII is allowed to be located at $x=0$ or $x=D$. This simplifies the summary, which we present in the following theorem. We omit the proof since it is easier than that of Theorem 4.4 .

THEOREM 4.6. Assume that $A^{\prime}(x)=0$ for $0<x<D$. The different types of solutions in the clarification zone, CI, etc., are given by Lemma 4.2 and in the thickening zone there are three possible types:

TI. $u_{r}(x)=u_{+}<u_{m}, x \in(0, D)$, with $\Phi_{\text {thick }}<\Phi_{\mathrm{sb}}$.

TII. $u_{r}(x)=u_{r}(D)>u_{M}, x \in(0, D)$, with $\Phi_{\text {thick }}>\Phi_{\mathrm{sb}}$.

TIII. $u_{r}(x)=\left\{\begin{array}{ll}u_{m}, & 0<x<x_{1} \\ u_{M}, & x_{1}<x<D\end{array}\right.$ for some $x_{1} \in[0, D]$ with $\Phi_{\text {thick }}=\Phi_{\mathrm{sb}}$.

The classification of the steady-state solutions is as follows.

$\star S<\Phi_{\text {lim }}$. CI, $u_{e}=0, \Phi_{\text {thick }}=S$, and $u_{u}=S / Q_{u}$. In the thickening zone, the following holds:

\begin{tabular}{|l||c|c|c|}
\hline & $S<\Phi_{\mathrm{sb}}$ & $S=\Phi_{\mathrm{sb}}$ & $S>\Phi_{\mathrm{sb}}$ \\
\hline \hline $0<u_{f}<u_{M}$ & & $\emptyset$ & $\emptyset$ \\
\cline { 1 - 3 }$u_{M} \leq u_{f} \leq u_{\max }$ & \multirow{2}{*}{ TI } & TIII & TII, $u_{M}<u_{+}<u_{f}<u_{\max }$ \\
\cline { 3 - 4 }
\end{tabular}

S $\underline{S=\Phi_{\text {lim }}}$. CI or CII, $u_{e}=0, \Phi_{\text {thick }}=S$ and $u_{u}=S / Q_{u}$. In the thickening zone, the following holds: 


\begin{tabular}{|l||c|c|c|}
\hline & $S<\Phi_{\mathrm{sb}}$ & $S=\Phi_{\mathrm{sb}}$ & $S>\Phi_{\mathrm{sb}}$ \\
\hline \hline $0<u_{f}<u_{m}$ & TI, $u_{+}=u_{f}=u_{z}$ & $\emptyset$ & \\
\cline { 1 - 3 }$u_{m} \leq u_{f} \leq u_{M}$ & & TIII, & \\
\cline { 1 - 3 }$u_{M}<u_{f} \leq u_{\max }$ & $\emptyset$ & $\emptyset$ & TII, $u_{+}=u_{f}=u_{z}$ \\
\hline
\end{tabular}

$\star S>\Phi_{\text {lim }}$. CIII, $\Phi_{\text {thick }}=\Phi_{\text {lim }}, \Phi_{\text {clar }}=S-\Phi_{\text {lim }}, u_{e}=\Phi_{\text {clar }} / Q_{e}>0, u_{u}=$ $\Phi_{\lim } / Q_{u}$. In the thickening zone, the following holds:

\begin{tabular}{|l||c|c|c|}
\hline & $\Phi_{\lim }<\Phi_{\mathrm{sb}}$ & $\Phi_{\lim }=\Phi_{\mathrm{sb}}$ & $\Phi_{\lim }>\Phi_{\mathrm{sb}}$ \\
\hline \hline $0<u_{f}<u_{m}$ & TI, $u_{-}=u_{+}=u_{f}$ & $\emptyset$ & \multirow{2}{*}{} \\
\hline$u_{f}=u_{m}$ & & TIII, $u_{-}=u_{m}$ & \multirow{2}{*}{$\emptyset$} \\
\cline { 3 - 4 }$u_{m}<u_{f} \leq u_{M}$ & $\emptyset$ & $\begin{array}{c}u_{r}(x) \equiv u_{M}, \\
u_{f} \leq u_{-} \leq u_{M}\end{array}$ & \\
\cline { 3 - 4 } & & $\emptyset$ & TII, \\
$u_{M}<u_{f} \leq u_{\max }$ & & $\emptyset$ & $u_{-}=u_{+}=u_{f}$ \\
\hline
\end{tabular}

The tables and the equation $f\left(u_{+}, 0\right)=g\left(u_{-}, 0\right)+s$ determine the concentrations $u_{-} \leq u_{+}$uniquely.

Note that the sludge blanket can be located anywhere when $A$ is constant.

4.3. Optimal steady-state operation. The main purpose of the settler is that it should produce a zero effluent concentration and a high underflow concentration. An additional purpose in waste water treatment is that the settler should be a buffer of mass, since a part of the biological sludge of the underflow is recycled within the plant. This can be achieved by adjusting $Q_{u}$ so that a steady-state solution with a discontinuity arises. Furthermore, the behavior of the settler should be rather insensitive to small variations in $u_{f}$ or in the $Q$-flows.

Chancelier, de Lara, and Picard [7] show that a discontinuity in the clarification zone (corresponding to the one of type CII) satisfies an algebraic-differential system and point out how it may be controlled dynamically by feedback. A stationary solution with type CII occurs only if $S=\Phi_{\text {lim }}$, see Theorems 4.4 and 4.6. Lemma 4.2 gives that $\Phi_{\text {clar }}=0$ independently of the location $x_{0} \in(-H, 0)$ of the discontinuity. Hence, the values of $Q_{e}$ and $u_{u}$ are independent of $x_{0}$. A small change in any $Q$-flow or $u_{f}$ will cause an inequality $\left(S \lessgtr \Phi_{\text {lim }}\right)$ instead, which either yields a zero concentration in the clarification zone or yields an overflow of sludge at steady state. Note that this is the case regardless of the shape of the clarification zone. This is probably the reason why one normally tries to adjust $Q_{u}$ so that, instead, a sludge blanket in the thickening zone arises. For a settler with constant $A$, a stationary sludge blanket is possible only if $S=\Phi_{\mathrm{sb}}$; see Theorem 4.6. Again, any small disturbance will cause an inequality $\left(S \lessgtr \Phi_{\mathrm{sb}}\right)$, which implies that the sludge blanket will increase or decrease dynamically with constant speed (after a transient).

According to Theorem 4.4, this problem can be avoided in a settler with $A^{\prime}(x)<0$ in the thickening zone by letting

$$
\Phi_{\mathrm{sb}}(D)<S<\Phi_{\lim }
$$

This is a sufficient condition for a steady-state solution of the combined type CI-TIII or TIIB (a sludge blanket at the feed level). Hence, (4.4) is a sufficient condition for 

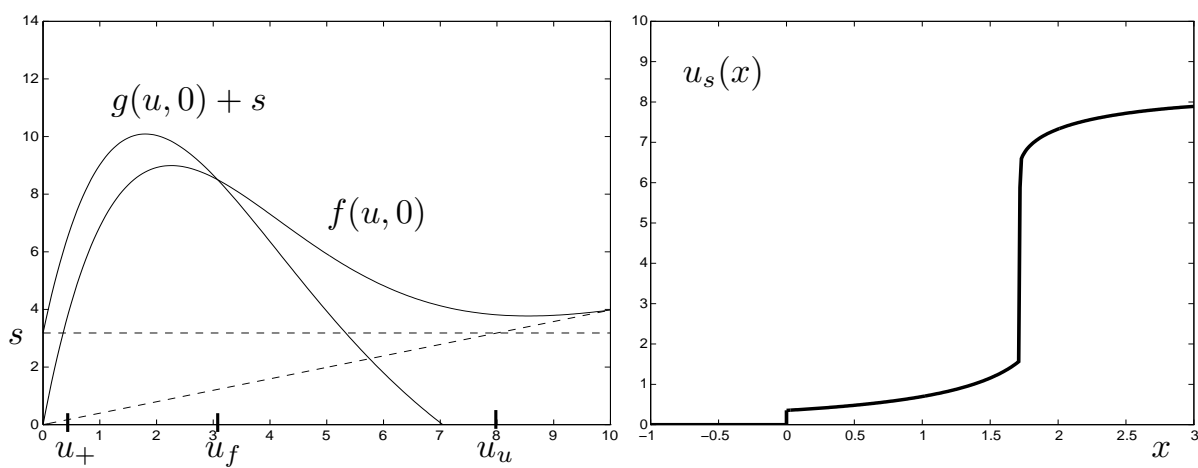

FIG. 4.2. A steady-state solution with a sludge blanket (CI-TIIIA) in a conical settler with $H=1 \mathrm{~m}, D=3 \mathrm{~m}, A(x)=\pi(20-5 x)^{2} \mathrm{~m}^{2}, Q_{f}=1300 \mathrm{~m}^{3} / \mathrm{h}, Q_{u}=500 \mathrm{~m}^{3} / \mathrm{h}, \Phi_{\mathrm{sb}}(1.71 \mathrm{~m})=$ $4000 \mathrm{~kg} / \mathrm{h}, u_{f}=3.08 \mathrm{~kg} / \mathrm{m}^{3}$, and $s=Q_{f} u_{f} / A(0)=3.18 \mathrm{~kg} /\left(\mathrm{m}^{2} \mathrm{~h}\right)$. Note how $u_{f}$ and $u_{u}$ can be obtained graphically (the inclined dashed line has the slope $v(0))$.

our definition of optimal operation. If, in addition,

$$
S<\Phi_{\mathrm{sb}}(0)
$$

holds, then the sludge blanket appears strictly below the feed level (TIII) by Theorem 4.4. An example of a steady-state solution in a conical settler for which (4.4) and (4.5) hold is given in Fig. 4.2. Note that the feed concentration $u_{f}$ is the unique intersection of the graphs of $f(\cdot, 0)$ and $g(\cdot, 0)+s$, since, with $u^{i}$ denoting an intersection,

$$
\begin{aligned}
& (v(0)+w(0)) u_{f}=\frac{Q_{u}+Q_{e}}{A(0)} u_{f}=\frac{Q_{f}}{A(0)} u_{f}=s \\
& \quad=f\left(u^{i}, 0\right)-g\left(u^{i}, 0\right)=\phi\left(u^{i}\right)+v(0) u^{i}-\left(\phi\left(u^{i}\right)-w(0) u^{i}\right)=(v(0)+w(0)) u^{i}
\end{aligned}
$$

and $v(0)+w(0)>0$.

A change in any variable such that (4.4) and (4.5) still hold will only cause a different depth of the sludge blanket at steady state. The interval $\left[\Phi_{\mathrm{sb}}(D), \Phi_{\mathrm{sb}}(0)\right]$ becomes larger the smaller $A(D)$ is and the larger $A(0)$ is and this should be of importance when designing a settler. Furthermore, for the cases of Theorem 4.4, note that $v(D)>\bar{v}$ is equivalent to $\Phi_{\mathrm{sb}}(D)<\Phi_{\mathrm{sb}}(0)$ and that $\bar{v}=-\phi^{\prime}\left(u_{\max }\right)$ is zero or close to zero in waste water treatment.

It is time to relate the terms underloaded, etc. to Theorem 4.4 .

- The settler is in optimal operation if (4.4) holds. This corresponds to the combination CI-TIII or TIIB (a sludge blanket at the feed level); see Fig. 4.3.

- The settler is underloaded if CI-TI holds, and a sufficient condition for this is that $S<\Phi_{\lim }$ and $S \leq \Phi_{\mathrm{sb}}(D)$ hold.

- The settler is overloaded if $u_{e}>0$, which is equivalent to $S>\Phi_{\text {lim; }}$; see Fig. 4.4 .

On the static control of the sludge blanket. Consider $Q_{f}$ and $u_{f}$ as given inputs, $Q_{u}$ as the control parameter and $Q_{e}, u_{u}$, and the depth $x_{1}$ of the sludge blanket as outputs. Therefore, we write out the dependence on $Q_{u}$, etc., e.g., $u_{M}\left(x, Q_{u}\right)$, and emphasize that this refers to steady-state solutions. The relations between the 

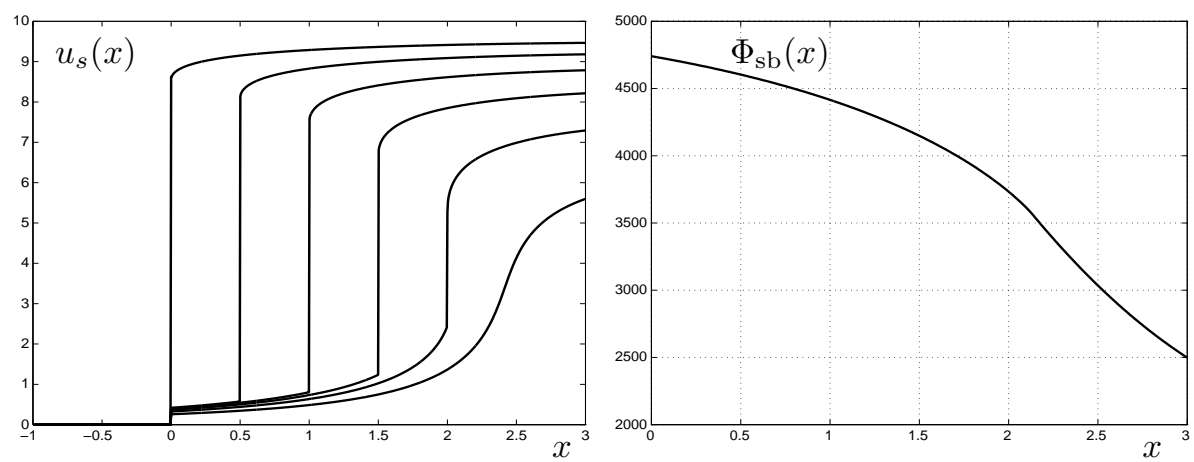

FIG. 4.3. Left: Steady-state solutions in optimal operation with the sludge blanket depths $x_{1}=$ $0 \mathrm{~m}$ (CI-TIIB), $x_{1}=0.5, \ldots, 2 \mathrm{~m}$ (CI-TIIIA), and $x_{1}=2.5 \mathrm{~m}$ (CI-TIIIB). The settler is conical with data as in Fig. 4.2; $Q_{u}=500 \mathrm{~m}^{3} / \mathrm{h}, Q_{f}=1300 \mathrm{~m}^{3} / \mathrm{h}$, and the feed concentrations are $u_{f}=3.65,3.54,3.40,3.19,2.87,2.33 \mathrm{~kg} / \mathrm{m}^{3}$. Right: The sludge blanket flux.
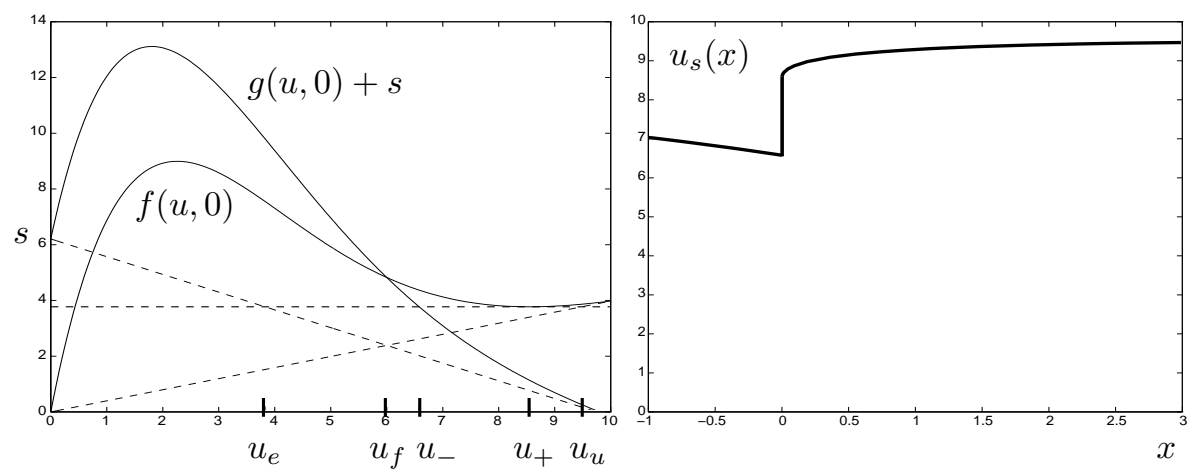

FIG. 4.4. An overloaded settler (CIII-TIIB, $\left.u_{m}(0)<u_{f}<u_{M}(0), u_{+}=u_{M}(0)\right)$ with the same data as in Fig. 4.2 except that $u_{f}=6 \mathrm{~kg} / \mathrm{m}^{3}$, which implies $s=Q_{f} u_{f} / A(0)=6.21 \mathrm{~kg} /\left(\mathrm{m}^{2} \mathrm{~h}\right)$, $\Phi_{\lim } / A(0)=f\left(u_{+}, 0\right)=3.77 \mathrm{~kg} /\left(\mathrm{m}^{2} \mathrm{~h}\right)$. Note how $u_{e}=3.82 \mathrm{~kg} / \mathrm{m}^{3}$ can be obtained graphically as the intersection of the dashed line with the slope $-w(0)$ and the horizontal line with value $f\left(u_{+}, 0\right)$, since $A(0) f\left(u_{+}, 0\right)=\Phi_{\lim }=S-\Phi_{\text {clar }}=A(0)\left(s-w(0) u_{e}\right)$.

relevant parameters of a steady-state solution of type CI-TIII are

$$
\begin{aligned}
Q_{f} u_{f} & =\Phi_{\mathrm{sb}}\left(x_{1}, Q_{u}\right)=Q_{u} u_{u}, \quad x_{1} \in(0, D), \\
Q_{f} & =Q_{u}+Q_{e} .
\end{aligned}
$$

In particular, this gives the interesting relation between the underflow concentration and the sludge blanket depth $x_{1}$ :

$$
u_{u}=\frac{\Phi_{\mathrm{sb}}\left(x_{1}, Q_{u}\right)}{Q_{u}}=\frac{A\left(x_{1}\right) \phi\left(u_{M}\left(x_{1}, Q_{u}\right)\right)}{Q_{u}}+u_{M}\left(x_{1}, Q_{u}\right) .
$$

For fixed $Q_{u}, u_{u}$ decreases with increasing $x_{1}$, because of (4.3) and the fact that $v\left(x_{1}\right)>\bar{v}$ in TIII. For example, consider the data of Fig. 4.3. If $Q_{u}=500 \mathrm{~m}^{3} / \mathrm{h}$ is kept fixed, then the corresponding underflow concentrations are $u_{u}=9.48,9.21,8.83$, $8.30,7.47,6.07 \mathrm{~kg} / \mathrm{m}^{3}$.

For given $Q_{f}$ and $u_{f}$, what is the value of $Q_{u}$ such that $Q_{e}$ and $u_{u}$ are maximized and such that the settler is still in optimal operation? The relations (4.6) give that 
$Q_{e}$ and $u_{u}$ are maximized precisely when $Q_{u}$ is minimized, and the following theorem says how low $Q_{u}$ can be.

THEOREM 4.7. Assume that $A^{\prime}(x)<0$ for $0<x<D$ and that $Q_{f}$ and $u_{f}$ are given. As long as

$$
Q_{u}>\bar{v} A(D)
$$

and

$$
Q_{u}>Q_{f}-\frac{A(0) \phi\left(u_{f}\right)}{u_{f}}
$$

hold, then

$$
\Phi_{\mathrm{sb}}\left(x_{1}, Q_{u}\right)=Q_{f} u_{f}, \quad 0<x_{1}<D
$$

defines implicitly the sludge blanket depth $x_{1}$ as an increasing function of the control parameter $Q_{u}$, corresponding to the solution-type CI-TIII of Theorem 4.4.

Proof. Theorem 4.4 gives that CI-TIII holds if (4.4) and (4.5) are satisfied, i.e., if

$$
\Phi_{\mathrm{sb}}\left(D, Q_{u}\right)<S=Q_{f} u_{f}<\min \left(\Phi_{\mathrm{sb}}\left(0, Q_{u}\right), \Phi_{\lim }\left(Q_{u}\right)\right)
$$

is satisfied. To verify this, first note that $\Phi_{\mathrm{sb}}\left(D, Q_{u}\right)<\Phi_{\mathrm{sb}}\left(0, Q_{u}\right) \Leftrightarrow v\left(D, Q_{u}\right)>\bar{v} \Leftrightarrow$ (4.7). Second, by the definition of $\Phi_{\text {lim }}$, we have

$$
\Phi_{\lim }\left(Q_{u}\right)= \begin{cases}A(0) f\left(u_{f}, 0, Q_{u}\right)<\Phi_{\mathrm{sb}}\left(0, Q_{u}\right), & u_{f} \in\left[0, u_{m}\left(0, Q_{u}\right)\right), \\ \Phi_{\mathrm{sb}}\left(0, Q_{u}\right), & u_{f} \in\left[u_{m}\left(0, Q_{u}\right), u_{M}\left(0, Q_{u}\right)\right], \\ A(0) f\left(u_{f}, 0, Q_{u}\right)>\Phi_{\mathrm{sb}}\left(0, Q_{u}\right), & u_{f} \in\left(u_{M}\left(0, Q_{u}\right), u_{\max }\right] .\end{cases}
$$

The inequality (4.8) is equivalent to $S<A(0) f\left(u_{f}, 0, Q_{u}\right)$, which together with (4.9) and (4.11) implies (4.10).

Differentiating $\Phi_{\mathrm{sb}}\left(x_{1}, Q_{u}\right)=Q_{f} u_{f}$ gives

$$
\frac{d Q_{u}}{d x_{1}}=-\frac{\partial \Phi_{\mathrm{sb}} / \partial x_{1}}{\partial \Phi_{\mathrm{sb}} / \partial Q_{u}}=-\frac{A^{\prime}\left(x_{1}\right) \phi\left(u_{M}\left(x_{1}, Q_{u}\right)\right)}{u_{M}\left(x_{1}, Q_{u}\right)}>0, \quad x_{1} \in(0, D),
$$

because $\Phi_{\mathrm{sb}}\left(D, Q_{u}\right)<\Phi_{\mathrm{sb}}\left(x_{1}, Q_{u}\right)<\Phi_{\mathrm{sb}}\left(0, Q_{u}\right)$ implies, by Lemma 4.3, $v\left(x_{1}, Q_{u}\right)>$ $\bar{v}$, which gives $u_{M}\left(x_{1}, Q_{u}\right)<u_{\max }$ and thus $\phi\left(u_{M}\left(x_{1}, Q_{u}\right)\right)>0$.

Consider the conical settler with data as in Fig. 4.2. Assume that $Q_{f}=1300 \mathrm{~m}^{3} / \mathrm{h}$ and that we want to keep the sludge blanket level at the depth $1.5 \mathrm{~m}$ at steady state. Fig. 4.5 shows the correspondence between $u_{f}$ and $Q_{u}$ given by (4.9). Note that (4.7), $Q_{u}>11.3 \mathrm{~m}^{3} / \mathrm{h}$, is not a severe restriction. (4.8) imposes no restriction at all in this case since the right-hand side is always less than $Q_{u}$ for each given $u_{f}$.

On the design of a settler. Under the given assumptions on sedimentation, the analysis in this paper yields that it is the cross-sectional area $A(x)$, the batch settling flux $\phi(u)$, and the underflow rate $Q_{u}$ that influence the behavior of the settler in optimal operation. Given $\phi(u)$ and the range of $Q_{u}$, the shape of the settler in the thickening zone, i.e., $A(x)$ for $0<x<D$, can be determined by means of the following information.

First, for an optimal steady-state solution, (4.7) and (4.8) yield that $A(0)$ should be large and $A(D)$ small. 


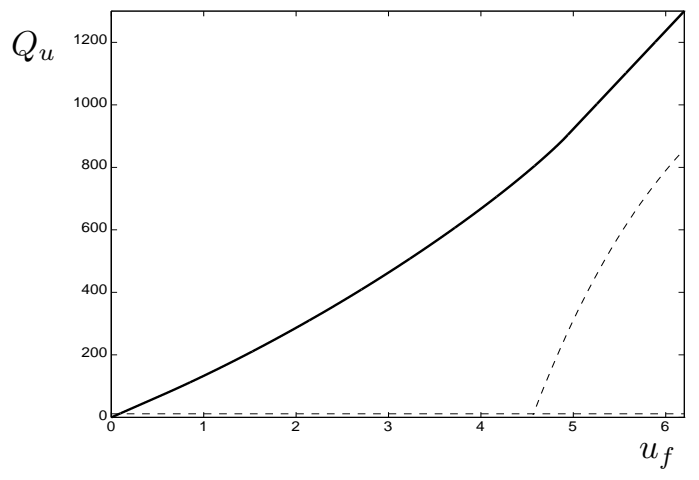

FIG. 4.5. An illustration of Theorem 4.7. The correspondence between $Q_{u}$ and $u_{f}=$ $\Phi_{\mathrm{sb}}\left(1.5, Q_{u}\right) / Q_{f}$ for obtaining the sludge blanket at the depth $1.5 \mathrm{~m}$. The horizontal dashed line lies on $\bar{v} A(3)=11.3 \mathrm{~m}^{3} / \mathrm{h}$, and the dashed curve is the right-hand side of (4.8) as a function of $u_{f}$. Note that $0 \leq Q_{u} \leq Q_{f}=1300 \mathrm{~m}^{3} / \mathrm{h}$

Second, assume that $Q_{u}$ is fixed. (In some waste water treatment plants $Q_{u}$ can only be adjusted at certain time points.) The shape of the settler influences the sensitivity of the sludge blanket depth $x_{1}$ for small variations in $S=Q_{f} u_{f}$. This follows from (4.9) and can be motivated qualitatively as follows. In a region where $A^{\prime}(x)$ is close to zero, $u_{M}(x)$ is almost constant; hence, $\Phi_{\mathrm{sb}}(x)$ is almost constant, and a small step change in $S=Q_{f} u_{f}$ will imply a large change in $x_{1}$ at the new steady state. On the contrary, $x_{1}$ is rather insensitive to small changes in $S=Q_{f} u_{f}$ if $A^{\prime}\left(x_{1}\right) \ll 0$, since $\Phi_{\mathrm{sb}}(x)$ is then more rapidly decreasing. However, $\Phi_{\mathrm{sb}}(x)=$ $A(x) f\left(u_{M}(x), x\right)$ depends not only on $A(x)$ but also on the batch settling flux, clearly illustrated in Fig. 4.3 (right) (cf. the graph of $A(x)$, which is a parabola for a conical settler).

Generally, the reasoning in the last paragraph should be applied to all relevant values of $Q_{u}$. In other words, the study of $\Phi_{\mathrm{sb}}\left(x, Q_{u}\right)=A(x) f\left(u_{M}\left(x, Q_{u}\right), x, Q_{u}\right)$ can give much information on how to form the shape of the settler in the thickening zone, given that the settler should normally have a specific sludge blanket depth and keep a certain mass of sludge.

5. Numerical simulations. The theoretical investigations in the previous sections will be supported here by numerical simulations. We shall present an algorithm using Godunov's [20] method as a basis. The numerical fluxes in Godunov's method are obtained by averaging analytical solutions of Riemann problems, in which the initial data consist of a single step. If the initial data are approximated by a piecewise constant function, such Riemann problems arise locally at the discontinuities of this initial value function. If the cross-sectional area is constant in a neighborhood of these discontinuities, the analytical solution of the Riemann problem can be used to obtain the averages forming the Godunov fluxes exactly. The updates of the boundary values are done by using the explicit formulas for the boundary concentrations given in [11] and referred to in the proof of Theorem 3.1. No convergence proof of the algorithm is presented.

A numerical algorithm. Divide the $x$-axis by $n$ grid points equally distributed, such that $x=-H$ and $x=D$ are located halfway between the first two and the last two grid points, respectively. Let the integer $i$ stand for space grid point at $x=x_{i}$, the integer $j$ for the time marching, and $U_{i}^{j}$ for the corresponding concentration. The 
feed source is assumed to be located at the grid point, denoted $i=m$, closest to $x=0$. The distance between two grid points is thus $\Delta=x_{i+1}-x_{i}=(H+D) /(n-2)$, and the grid point $m=\operatorname{round}(H / \Delta+3 / 2)$ is nearest to the feed level. The length of the time step is denoted by $\tau$. According to the motivation above, we make the assumption that the cross-sectional area is piecewise constant between two grid points, that is, for fixed $j$

$$
A(x)=A_{i+1 / 2}^{j}, \quad x_{i} \leq x<x_{i+1}, \quad i=1, \ldots, n,
$$

and we define

$$
A_{i}^{j}=\frac{A_{i+1 / 2}^{j}+A_{i-1 / 2}^{j}}{2}, \quad i=2, \ldots, n-1
$$

Let

$$
\tilde{u}^{j}(x, j \tau)=U_{i}^{j}, \quad x_{i} \leq x<x_{i+1}, \quad i=1, \ldots, n,
$$

be piecewise constant initial data at time $t=j \tau$ and let $\tilde{u}^{j}(x, t)$ be the analytical solution built up of solutions of parallel Riemann problems. Thus $\tilde{u}(x, t)$ satisfies $u_{t}+g(u)_{x}=0$ in the clarification zone and $u_{t}+f(u)_{x}=0$ in the thickening zone. Define the averages

$$
U_{i}^{j+1}=\frac{1}{\Delta A_{i}^{j}} \int_{x_{i}-\Delta / 2}^{x_{i}+\Delta / 2} A(x) \tilde{u}^{j}(x,(j+1) \tau) d x, \quad i=2, \ldots, n-1 .
$$

The conservation law on integral form is, for example, in the clarification zone

$$
\begin{aligned}
\frac{d}{d t} \int_{x_{i}-\Delta / 2}^{x_{i}+\Delta / 2} A(x) \tilde{u}^{j}(x, t) d x & =A_{i-1 / 2}^{j} g\left(\tilde{u}^{j}\left(x_{i}-\frac{\Delta}{2}, t\right), x_{i}-\frac{\Delta}{2}\right) \\
- & A_{i+1 / 2}^{j} g\left(\tilde{u}^{j}\left(x_{i}+\frac{\Delta}{2}, t\right), x_{i}+\frac{\Delta}{2}\right), \quad i=2, \ldots, m-1 .
\end{aligned}
$$

An analogous equation holds for the thickening zone and the flux function $f$ and at the grid point $m$ the source term is added on the right-hand side in a natural way. If $\tau$ satisfies

$$
\frac{\tau}{\Delta}<\min \left(\frac{1}{\max _{u \in\left[0, u_{\max }\right]}\left|\partial_{u} f(u, x)\right|}, \frac{1}{\max _{\substack{u \in\left[0, u_{\max }\right] \\ x \in[-H, 0]}}\left|\partial_{u} g(u, x)\right|}\right)
$$

then the solution $\tilde{u}$ is constant on the line segments $j \tau \leq t<(j+1) \tau, x=x_{i}+\Delta / 2$, $i=1, \ldots, n-1$, which is necessary for forming the Godunov fluxes. Integrating (5.1) (and the analogous equations for the thickening zone and for the grid point $i=m$ ) from $j \tau$ to $(j+1) \tau$ and dividing by $\Delta A_{i}^{j}$, the following scheme is obtained for the 
grid points $i=2, \ldots, n-1$ :

$$
\begin{aligned}
U_{i}^{j+1} & =U_{i}^{j}+\frac{\tau}{\Delta A_{i}^{j}}\left(A_{i-1 / 2}^{j} G_{i-1 / 2}^{j}-A_{i+1 / 2}^{j} G_{i+1 / 2}^{j}\right), & & i=2, \ldots, m-1, \\
U_{m}^{j+1} & =U_{m}^{j}+\frac{\tau}{\Delta A_{i}^{j}}\left(A_{m-1 / 2}^{j} G_{m-1 / 2}^{j}-A_{m+1 / 2}^{j} F_{m+1 / 2}^{j}+S^{j}\right), & & i=m, \\
U_{i}^{j+1} & =U_{i}^{j}+\frac{\tau}{\Delta A_{i}^{j}}\left(A_{i-1 / 2}^{j} F_{i-1 / 2}^{j}-A_{i+1 / 2}^{j} F_{i+1 / 2}^{j}\right), & & i=m+1, \ldots, n-1,
\end{aligned}
$$

where Godunov's numerical flux is (analogously for $F$ and $f$ )

$$
G_{i-1 / 2}^{j}= \begin{cases}\min _{v \in\left[U_{i-1}^{j}, U_{i}^{j}\right]} g\left(v, x_{i}-\frac{\Delta}{2}\right) & \text { if } U_{i-1}^{j} \leq U_{i}^{j}, \\ \max _{v \in\left[U_{i}^{j}, U_{i-1}^{j}\right]} g\left(v, x_{i}-\frac{\Delta}{2}\right) & \text { if } U_{i-1}^{j}>U_{i}^{j},\end{cases}
$$

and $S^{j}=Q_{f} u_{f}^{j}$, which is an average over $j \tau<t<(j+1) \tau$.

Then the boundary values (grid points 1 and $n$ ) and the outputs $u_{e}$ and $u_{u}$ are updated according to, cf. [11],

$$
\begin{aligned}
& U_{1}^{j+1}= \begin{cases}U_{2}^{j+1} & \text { if } g\left(U_{2}^{j+1},-H\right) \leq 0, \\
0 & \text { if } g\left(U_{2}^{j+1},-H\right)>0,\end{cases} \\
& u_{e}^{j+1}=U_{1}^{j+1}-\frac{\phi\left(U_{1}^{j+1}\right)}{w(-H)}, \\
& U_{n}^{j+1}= \begin{cases}U_{n-1}^{j+1} & \text { if } U_{n-1}^{j+1} \in\left[0, u_{m}(D)\right) \cup\left(u_{M}(D), u_{\max }\right], \\
u_{M} & \text { if } U_{n-1}^{j+1} \in\left[u_{m}(D), u_{M}(D)\right],\end{cases} \\
& u_{u}^{j+1}=U_{n}^{j+1}+\frac{\phi\left(U_{n}^{j+1}\right)}{v(D)} .
\end{aligned}
$$

Two simulations. In Figs. 5.1 and 5.2 the results of two simulations are shown. The settler is conical with $H=1 \mathrm{~m}, D=3 \mathrm{~m}, A(x)=\pi(20-5 x)^{2} \mathrm{~m}^{2}$. The flows $Q_{f}=1300 \mathrm{~m}^{3} / \mathrm{h}$ and $Q_{u}=500 \mathrm{~m}^{3} / \mathrm{h}$ are kept constant. These are the same data as in the examples shown in Figs. 4.2, 4.3, and 4.4.

The initial value function in Fig. 5.1 is the steady-state solution shown in Fig. 4.2, which corresponds to $u_{f}=3.18 \mathrm{~kg} / \mathrm{m}^{3}$. At $t=0 \mathrm{~h}$, the feed concentration is set to the larger value $u_{f}=6 \mathrm{~kg} / \mathrm{m}^{3}$. The extra amount of sludge fed into the settler implies that the sludge blanket, originally at the depth of $1.7 \mathrm{~m}$, rises, and after two hours it reaches the feed point. After that, a large discontinuity rises in the clarification zone, and the steady-state solution of Fig. 4.4 will be obtained asymptotically.

The second simulation, see Fig. 5.2, demonstrates some of the steady-state solutions shown in Fig. 4.3 (left). The initial value function is a steady-state solution with a sludge blanket at $1.5 \mathrm{~m}$ and with the sludge blanket flux $\Phi_{\mathrm{sb}}(1.5)=4149 \mathrm{~kg} / \mathrm{h}$ corresponding to $u_{f}=3.19 \mathrm{~kg} / \mathrm{m}^{3}$. At $t=0 \mathrm{~h}$, the feed concentration is set to the lower value $2.33 \mathrm{~kg} / \mathrm{m}^{3}$. Then, already at $t \approx 3 \mathrm{~h}$, the rightmost steady-state solution in Fig. 4.3 (left) is formed. This solution is continuous, although we have defined the sludge blanket at the depth of $2.5 \mathrm{~m}$, which is the depth where the concentration is $u_{\text {infl. }}$. At $t=4 \mathrm{~h}$, the feed concentration is changed to $2.87 \mathrm{~kg} / \mathrm{m}^{3}$, which implies that a new steady state is formed with a sludge blanket at the depth of $2 \mathrm{~m}$.

6. Conclusions. The dynamic behavior of continuous sedimentation in a settler with varying cross-sectional area has been analyzed with the following outcomes: 

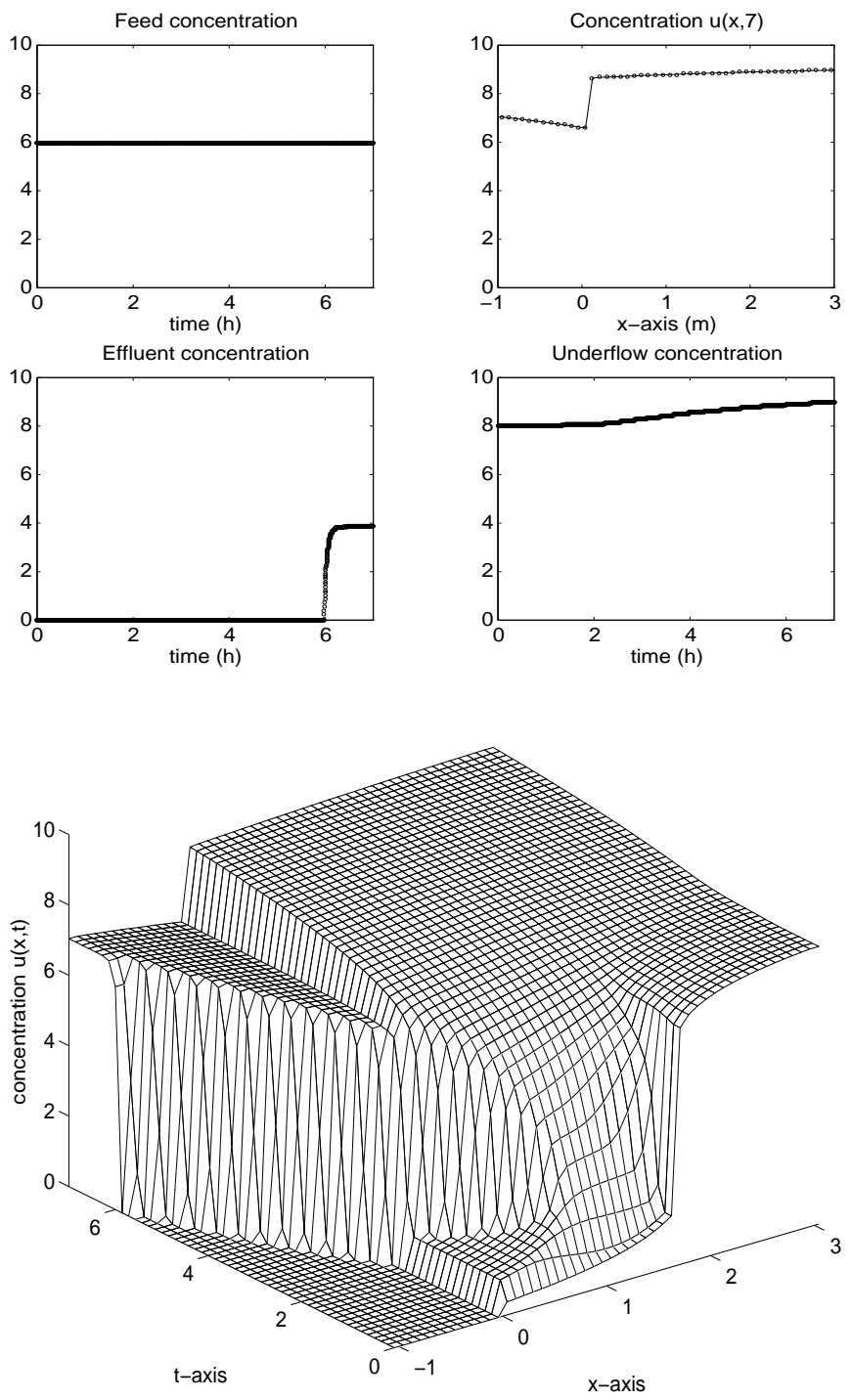

FIG. 5.1. A dynamic simulation with initial data from Fig. 4.2 and with the asymptotic solution as in Fig. 4.4. The number of grid points is $n=50$.

- a theorem on existence and uniqueness;

- a numerical algorithm.

The steady-state behavior has been analyzed with the following outcomes:

- a complete classification of the steady-state solutions when $A^{\prime}(x) \leq 0$ in the thickening zone $(A(x)$ is arbitrary in the clarification zone);

- explicit formulas on the static control of the settler in optimal operation, by using $Q_{u}$ as a control parameter;

- an explicit formula for the underflow concentration as a function of the sludge blanket depth;

- a discussion on the design of a settler; the cross-sectional area's impact on the settler behavior.

Appendix A. Proof of Theorem 4.4. In the proofs below we shall always use the jump and entropy conditions for scalar conservation laws with continuous flux 

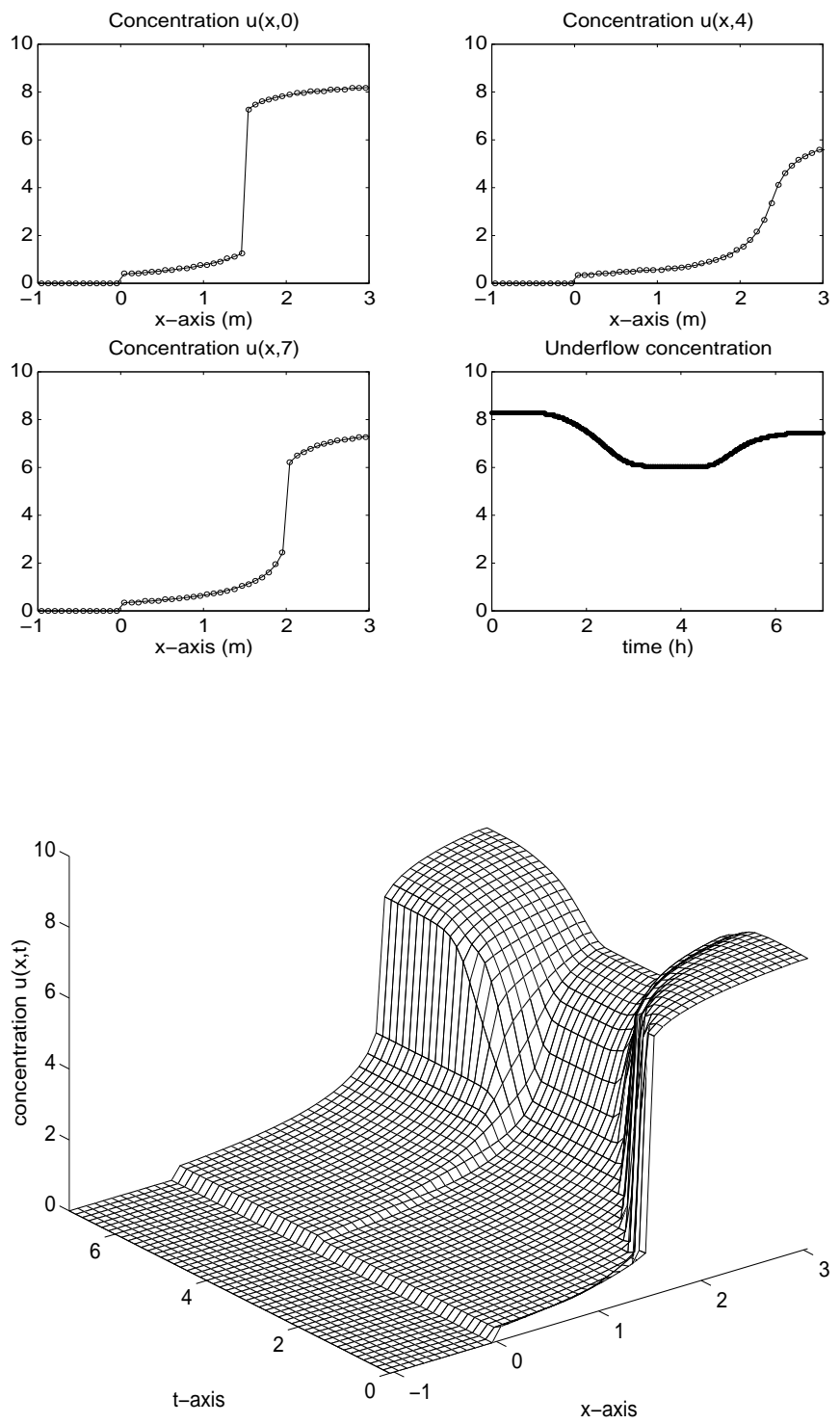

FIG. 5.2. A dynamic simulation showing three steady-state solutions (at $t=0,4,7 \mathrm{~h}$ ) of Fig. 4.3 (left).

function. For a stationary discontinuity at $x$, the jump condition is simply $f\left(u^{-}, x\right)=$ $f\left(u^{+}, x\right)$, where $u^{ \pm}$are the concentrations to the left and right of the discontinuity. The entropy condition reads

$$
\frac{f(\tilde{u}, x)-f\left(u^{-}, x\right)}{\tilde{u}-u^{-}} \geq 0 \quad \text { for all } \tilde{u} \text { between } u^{-} \text {and } u^{+} .
$$

The following lemma considers the solutions of the equation $f\left(u_{+}, 0\right)=g\left(u_{-}, 0\right)+$ $s$. Note that multiplying by $A(0)$ this equation becomes $S=\Phi_{\text {thick }}+\Phi_{\text {clar }}$.

LEMMA A.1. Necessary conditions on the concentrations just above and below the feed inlet at steady state are $u_{-} \leq u_{+}$and 
$0<u_{f} \leq u_{m}(0)$

- $s<f\left(u_{f}, 0\right): u_{-}=0, u_{+}$is uniquely determined by $f\left(u_{+}, 0\right)=s, 0<u_{+}<$ $u_{f}$.

- $s=f\left(u_{f}, 0\right):\left(u_{f}=u_{z}(0)\right),\left(u_{-}, u_{+}\right)=\left(0, u_{f}\right)$ or $u_{-}=u_{+}=u_{f}$. The possibility $\left(u_{-}, u_{+}\right)=\left(u_{m}(0), u_{M}(0)\right)$ holds only if $u_{f}=u_{m}(0)$.

- $s>f\left(u_{f}, 0\right): u_{-}=u_{+}=u_{f}>u_{z}(0)$.

$u_{m}(0)<u_{f}<u_{M}(0):$

$\bullet s<f\left(u_{M}(0), 0\right): u_{-}=0, u_{+}$is uniquely determined by $f\left(u_{+}, 0\right)=s, 0<$ $u_{+}<u_{m}(0)$.

- $s=f\left(u_{M}(0), 0\right):\left(u_{f}<u_{z}(0)<u_{M}(0)\right),\left(u_{-}, u_{+}\right)=\left(0, u_{m}(0)\right),\left(u_{-}, u_{+}\right)=$ $\left(0, u_{M}(0)\right)$ or $\left(u_{-}, u_{+}\right)=\left(u_{z}(0), u_{M}(0)\right)$.

- $s>f\left(u_{M}(0), 0\right): u_{-}>u_{z}(0)$ is uniquely determined by $g\left(u_{-}, 0\right)=$ $f\left(u_{M}(0), 0\right)-s$ and satisfies $u_{f}<u_{-}<u_{M}(0), u_{+}=u_{M}(0)$.

$u_{M}(0) \leq u_{f} \leq u_{\max }:$

$\bullet s<f\left(u_{M}(0), 0\right): u_{-}=0, u_{+}$is uniquely determined by $f\left(u_{+}, 0\right)=s, 0<$ $u_{+}<u_{m}(0)$.

- $s=f\left(u_{M}(0), 0\right):\left(u_{-}, u_{+}\right)=\left(0, u_{m}(0)\right)$ or $\left(u_{-}, u_{+}\right)=\left(0, u_{M}(0)\right)$.

- $f\left(u_{M}(0), 0\right)<s<f\left(u_{f}, 0\right):$ (necessarily $\left.u_{M}(0)<u_{f}<u_{\max }\right), u_{-}=0, u_{+}$ is uniquely determined by $f\left(u_{+}, 0\right)=s, u_{M}(0)<u_{+}<u_{f}$.

- $s=f\left(u_{f}, 0\right):\left(u_{-}, u_{+}\right)=\left(0, u_{f}\right)$ or $u_{-}=u_{+}=u_{f}=u_{z}(0)$.

- $s>f\left(u_{f}, 0\right): u_{-}=u_{+}=u_{f}>u_{z}(0)$.

Proof. See section 9 in [11].

Proof of Lemma 4.2. $u_{l}(x)$ is a piecewise smooth solution of the implicit equation

$$
A(x) g\left(u_{l}(x), x\right)=-\Phi_{\text {clar }}, \quad-H<x<0,
$$

where $g(u, x)=\phi(u)-w(x) u$ and $\Phi_{\text {clar }} \geq 0$ is a constant. In a neighborhood of points where $\partial_{u} g\left(u_{l}(x), x\right) \neq 0,($ A.1) implies

$$
u_{l}^{\prime}(x)=-\frac{A^{\prime}(x) \phi\left(u_{l}(x)\right)}{A(x) \partial_{u} g\left(u_{l}(x), x\right)} .
$$

Lemma 4.1 gives the possible boundary limits at $x=-H$, underlined below.

Assume that $u_{l}(-H)=0$, which means that $u_{l}(x)$ is smooth in a right neighborhood of $-H$ and $\overline{(\mathrm{A} .2) \text { gives }} u_{l}^{\prime}(x)=0$ in this neighborhood. It also follows directly that $\Phi_{\text {clar }}=-A(-H) g(0,-H)=0$. Either $u_{l}(x) \equiv 0$ or there is a discontinuity at some $x_{0} \in(-H, 0)$ with left value 0 and right value $u_{z}\left(x_{0}\right)$. By definition of $u_{z}$, $\partial_{u} g\left(u_{z}(x), x\right)<0$, hence, $u_{l}(x)$ is smooth to the right of the discontinuity satisfying

$$
\begin{aligned}
& A(x) g\left(u_{l}(x), x\right)=-\Phi_{\text {clar }}=0, \quad x_{0}<x<0, \\
& u_{l}\left(x_{0}+\right)=u_{z}\left(x_{0}\right),
\end{aligned}
$$

which has the unique solution $u_{l}(x)=u_{z}(x), x_{0}<x<0$. The uniqueness follows from the basic uniqueness theorem for ordinary differential equations since the solution satisfies (A.2) with the right-hand side at least Lipschitz continuous.

Assume that $u_{l}(-H)=u_{z}(-H)$. Then $\Phi_{\text {clar }}=0$ and (A.3) with $x_{0}=-H$ gives $u_{l}(x) \equiv u_{z}(x)$. We have proved CI and CII.

Assume that $u_{l}(-H)>u_{z}(-H)$. Then $\Phi_{\text {clar }}=-A(-H) g\left(u_{l}(-H),-H\right)>0$. Using this fact together with $g\left(u_{z}(x), x\right) \equiv 0$ and that $u_{l}(x)$ satisfies (A.1) we get

$$
A(x)\left(g\left(u_{l}(x), x\right)-g\left(u_{z}(x), x\right)\right)=-\Phi_{\text {clar }}, \quad-H<x<0 .
$$


For every $x \in(-H, 0)$ there exists a $\xi(x)$ between $u_{l}(x)$ and $u_{z}(x)$ such that

$$
\partial_{u} g(\xi(x), x)\left(u_{l}(x)-u_{z}(x)\right)=\frac{-\Phi_{\text {clar }}}{A(x)} .
$$

Since $\partial_{u} g(\xi(x), x)<0$ for $x$ in a right neighborhood of $-H$, it follows that $u_{l}(x)>$ $u_{z}(x)$ in this neighborhood. However, since the right-hand side of (A.4) is $<0$, it follows that $u_{l}(x)>u_{z}(x)$ for all $x \in(-H, 0)$. Finally, no discontinuity is possible with left value $>u_{z}(x)$. Item CIII is proved. Finally, the claim on the sign of $u_{l}^{\prime}(x)$ follows from (A.2) for $u_{l}(x) \geq u_{z}(x)$ since in this case $\partial_{u} g\left(u_{l}(x), x\right)<0$ holds.

Proof of Lemma 4.3. $u_{r}(x)$ is a piecewise smooth solution of the implicit equation

$$
A(x) f\left(u_{r}(x), x\right)=\Phi_{\text {thick }}, \quad 0<x<D,
$$

where $f(u, x)=\phi(u)+v(x) u$ and $\Phi_{\text {thick }} \geq 0$ is a constant. In a neighborhood of points where $\partial_{u} f\left(u_{r}(x), x\right) \neq 0,($ A.5) implies

$$
u_{r}^{\prime}(x)=-\frac{A^{\prime}(x) \phi\left(u_{r}(x)\right)}{A(x) \partial_{u} f\left(u_{r}(x), x\right)} .
$$

Lemma 4.1 gives the possible boundary limits $u_{r}(D) \in\left[0, u_{m}(0)\right] \cup\left[u_{M}(0), u_{\max }\right]$. We shall underline the different cases. First, we conclude that $u_{r}(x) \equiv 0$ and $u_{r}(x) \equiv u_{\max }$ are the only two constant solutions of (A.5). Furthermore, the conditions $u_{r}\left(x_{0}\right)=0$ for any $x_{0} \in[0, D]$ and $u_{r}(x)$ continuous imply that $u_{r}(x) \equiv 0$ for $x \in(0, D)$ by uniqueness of solutions of (A.6) because $\partial_{u} f(0+, x)>0$ for $x \in(0, D)$. Since there is no possibility for a discontinuity with $u=0$ as left or right value, all other solutions satisfy $u_{r}(x)>0$ for $x \in(0, D)$.

Since $\partial_{u} f(\cdot, x)>0$ on $\left(0, u_{m}(x)\right]$ for every $x \in[0, D]$ we get

$$
\begin{gathered}
\frac{0<u_{r}(D) \leq u_{m}(D)}{A(x) f\left(u_{r}(x), x\right)=\Phi_{\text {thick }}=A(D) f\left(u_{r}(D), D\right)} \Longrightarrow \\
\leq A(D) f\left(u_{m}(D), D\right)=\Phi_{\mathrm{sb}}(D) \leq \Phi_{\mathrm{sb}}(x)=A(x) f\left(u_{m}(x), x\right), \quad 0<x<D \\
\Longleftrightarrow u_{r}(x) \leq u_{m}(x), \quad 0<x<D,
\end{gathered}
$$

which together with (A.6) implies that $u_{r}^{\prime}(x)>0 . u_{r}(x)=u_{m}(x)$ is impossible on any open interval, for substituting into (A.5) and differentiating gives $A^{\prime}(x) \phi\left(u_{m}(x)\right) \equiv 0$, which is a contradiction. Furthermore, no discontinuity is possible with right value strictly less than $u_{m}(x)$. TI is proved.

The boundary limits left are now $u_{M}(D) \leq u_{r}(D) \leq u_{\text {max }}$.

Assume that $\underline{u_{M}(D)<u_{r}(D)<u_{\max }}$. Then

$$
\Phi_{\text {thick }}=A(D) f\left(u_{r}(D), D\right)>A(D) f\left(u_{M}(D), D\right)=\Phi_{\mathrm{sb}}(D)
$$

because $\partial_{u} f(\cdot, x)>0$ on $\left(u_{M}(x), u_{\max }\right]$. Equation (A.6) says that $u_{r}^{\prime}(x)>0$ in a left neighborhood of $x=D$. Either $u_{r}(x)>u_{M}(x)$ for all $x \in(0, D)$, which implies $u_{M}(0) \leq u_{+} \equiv u_{r}(D)<u_{\max }$. Hence, $v(0)>\bar{v}$ and $\Phi_{\text {thick }} \geq \Phi_{\mathrm{sb}}(0)$, which gives TIIB. Otherwise, there exists an $x_{1} \in(0, D)$ with $u_{r}\left(x_{1}+0\right)=u_{M}\left(x_{1}\right)$, giving $\Phi_{\text {thick }}=\Phi_{\mathrm{sb}}\left(x_{1}\right)$. The property $u_{r}^{\prime}(x)>0$ for $x_{1}<x<D$ implies $u_{r}\left(x_{1}+0\right)=$ $u_{M}\left(x_{1}\right)<u_{\max }$, which in turn gives $v\left(x_{1}\right)>\bar{v}$. Then (4.3) gives $\Phi_{\mathrm{sb}}^{\prime}\left(x_{1}\right)<0$, hence, $\Phi_{\mathrm{sb}}(D)<\Phi_{\text {thick }}=\Phi_{\mathrm{sb}}\left(x_{1}\right)<\Phi_{\mathrm{sb}}(0)$, which determines $x_{1}$ uniquely for given $\Phi_{\text {thick }}$. We consider two subcases depending on $v\left(x_{1}\right) \lessgtr \overline{\bar{v}}$. 
First, if $\bar{v}<v\left(x_{1}\right)<\overline{\bar{v}}$, then $u_{r}\left(x_{1}+0\right)=u_{M}\left(x_{1}\right)>u_{\text {infl }}$. Assuming $u_{r}(x)=$ $u_{M}(x)$ in a left neighborhood of $x_{1}$, substituting into (A.5) and differentiating gives $A^{\prime}(x) \phi\left(u_{M}(x)\right) \equiv 0$, which is a contradiction, since $0<u_{M}(x)<u_{\max }$. If $u_{r}(x)$ were continuous at $x_{1}$ with $u_{r}(x)<u_{M}(x)$ in a left neighborhood of $x_{1}$, then $\partial_{u} f\left(u_{r}(x), x\right)<0$ and (A.6) implies $u_{r}^{\prime}(x) \rightarrow-\infty$ as $x / x_{1}$. Since $u_{M}^{\prime}(x)$ is finite, it follows that $u_{r}(x)>u_{M}(x)$ in a left neighborhood of $x_{1}$, contradicting the assumption. Thus, the only possibility is a discontinuity at $x_{1}$ with $u_{m}\left(x_{1}\right)$ as the left value and $u_{M}\left(x_{1}\right)$ as the right value. Replacing $D$ by $x_{1}$ in (A.7) implies $u_{r}(x)<u_{m}(x)$ for $0<x<x_{1}$. The case TIIIA is proved by concluding that (A.6) implies $u_{r}^{\prime}(x) \rightarrow \infty$ as $x \searrow x_{1}$.

Second, if $v\left(x_{1}\right) \geq \overline{\bar{v}}$, then $f\left(\cdot, x_{1}\right)$ is increasing and $u_{r}\left(x_{1}\right)=u_{M}\left(x_{1}\right)=u_{m}\left(x_{1}\right)=$

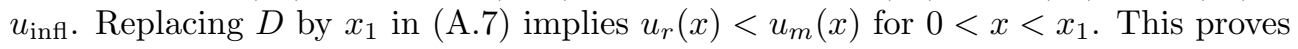
TIIIB.

Assume that $u_{M}(D)=u_{r}(D)<u_{\max }$. Using $D$ instead of $x_{1}$ in the reasoning two paragraphs above this yields a discontinuity at $x=D$, which implies $u_{r}(D)=$ $u_{m}(D)<u_{M}(D)$, a contradiction.

Assume that $u_{r}(D)=u_{\max }$. Either $u_{r}(x) \equiv u_{\max }$ for $0<x<D$ and then, since $\phi\left(u_{\max }\right)=0$,

$$
\Phi_{\text {thick }}=A(0) f\left(u_{\max }, 0\right)=Q_{u} u_{\max } \geq A(0) f\left(u_{M}(0), 0\right)=\Phi_{\mathrm{sb}}(0),
$$

which proves TIIA. With similar arguments as above, the only possibility left is a discontinuity at some $x_{1} \in(0, D)$ with $u_{m}\left(x_{1}\right)$ as left value and $u_{\max }$ as right value. Replacing $D$ by $x_{1}$ in (A.7) yields $u_{r}(x)<u_{m}(x)$ for $0<x<x_{1}$. Especially, $u_{+}<u_{m}(0)$ implies $\Phi_{\text {thick }}=A(0) f\left(u_{+}, 0\right)<A(0)\left(u_{m}(0), 0\right) \Phi_{\mathrm{sb}}(0)$, which contradicts (A.8).

LEMma A.2. When $A^{\prime}(x)<0, x \in(0, D)$, any steady-state solution satisfies $u_{+} \in\left[0, u_{m}(0)\right) \cup\left[u_{M}(0), u_{\max }\right]$.

Proof. This follows directly from Lemma 4.3.

Proof of Theorem 4.4. Recall that

$$
S \lessgtr \Phi_{\lim } \Longleftrightarrow s \lessgtr \begin{cases}f\left(u_{f}, 0\right), & u_{f} \in\left[0, u_{m}(0)\right] \cup\left[u_{M}(0), u_{\max }\right], \\ f\left(u_{M}(0), 0\right), & u_{f} \in\left(u_{m}(0), u_{M}(0)\right) .\end{cases}
$$

We shall generally assume that $v(D)>\bar{v}$ and only make some comments on the cases when $v(D) \leq \bar{v}$ since these are special cases (often empty cases) of the others because

$$
\begin{aligned}
v(D) \leq \bar{v} & \Longleftrightarrow \Phi_{\mathrm{sb}}(0)=\Phi_{\mathrm{sb}}(D) \quad \Longrightarrow \\
& v(0)<v(D) \leq \bar{v} \quad \Longrightarrow \quad u_{M}(0)=u_{\max } \quad \Longrightarrow \quad \Phi_{\lim } \leq \Phi_{\mathrm{sb}}(0)
\end{aligned}
$$

by the definition of $\Phi_{\text {lim }}$.

- $S<\Phi_{\text {lim }}$ : Lemma A.1 implies that $u_{-}=0$ and then Lemma 4.2 gives CI for the clarification zone. Hence, $S=\Phi_{\text {thick }}$.

$S=\Phi_{\text {thick }}<\Phi_{\mathrm{sb}}(0):$ Hence $s<\min \left(f\left(u_{M}(0), 0\right), f\left(u_{f}, 0\right)\right)$ holds and Lem$\overline{\text { ma A.1 implies } u_{+}} \equiv u_{r}(0)<\min \left(u_{f}, u_{m}(0)\right)$. Since $S=\Phi_{\text {thick }}<\Phi_{\mathrm{sb}}(0)$, Lemma 4.3 implies that the solution in the thickening zone is of type TI if $\Phi_{\text {thick }} \leq \Phi_{\mathrm{sb}}(D)$ and TIII if $\Phi_{\mathrm{sb}}(D)<\Phi_{\text {thick }}<\Phi_{\mathrm{sb}}(0)$.

$S=\Phi_{\text {thick }} \geq \Phi_{\mathrm{sb}}(0)$ : Then $f\left(u_{M}(0), 0\right) \leq s<\Phi_{\text {lim }} / A(0)$ holds, which implies that $\Phi_{\lim }=A(0) f\left(u_{f}, 0\right)$ and $u_{f}>u_{M}(0)$, otherwise this case is empty (e.g., when $v(D) \leq \bar{v})$. Lemma A.1 implies that either $u_{+}=u_{m}(0)$, which is 
impossible by Lemma A.2, or $u_{M}(0) \leq u_{+} \leq u_{f}<u_{\max }$. Lemma 4.3 then implies that the solution in the thickening zone is of type TIIB.

- $S=\Phi_{\text {lim }}$ : Lemma A.1 implies that $u_{-}=0$ or $u_{-}=u_{z}(0)$ and then Lemma 4.2 gives CI or CII are possible for the clarification zone, both with $\Phi_{\text {clar }}=0$. Hence, $S=\Phi_{\text {thick }}$.

$S=\Phi_{\text {thick }}<\Phi_{\mathrm{sb}}(0)$ : Thus, $s=f\left(u_{f}, 0\right)<f\left(u_{M}(0), 0\right)$, hence $u_{f}<u_{m}(0)$ and Lemma A.1 gives $u_{+}=u_{f}=u_{z}(0)$. Then Lemma 4.3 gives the possibilities TI or TIII according to the table, though only TI when $v(D) \leq \bar{v}$.

$S=\Phi_{\text {thick }}=\Phi_{\mathrm{sb}}(0)$ : This implies $s=f\left(u_{M}(0), 0\right)=\Phi_{\mathrm{lim}} / A(0)$, hence,

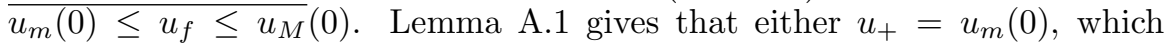
is impossible by Lemma A.2, or $u_{+}=u_{M}(0)$ with $u_{f} \leq u_{z}(0) \leq u_{M}(0)$. If $u_{+}=u_{M}(0)=u_{\max }$, i.e., $v(0) \leq \bar{v}$, then TIIA holds, otherwise TIIB.

$S=\Phi_{\text {thick }}>\Phi_{\mathrm{sb}}(0)$ : Then $f\left(u_{M}(0), 0\right)<s=\Phi_{\text {lim }} / A(0)$ holds, which implies that $\Phi_{\lim }=A(0) f\left(u_{f}, 0\right)$ and $u_{f}>u_{M}(0)$, otherwise this case is empty. Lemma A.1 implies that either $u_{+}=u_{m}(0)$, which is impossible by Lemma A.2, or $u_{+}=u_{f}=u_{z}(0)$. Lemma 4.3 then implies that the solution in the thickening zone is of type TIIA or B.

- $S>\Phi_{\text {lim }}$ : Lemma A.1 implies that $u_{-}>u_{z}(0)$ and that

$$
\begin{aligned}
u_{f} \in\left(0, u_{m}(0)\right] \cup\left[u_{M}(0), u_{\text {max }}\right] & \Longrightarrow u_{+}=u_{f} \\
& \Longrightarrow \Phi_{\text {thick }}=A(0) f\left(u_{f}, 0\right)=\Phi_{\text {lim }}, \\
u_{f} \in\left(u_{m}(0), u_{M}(0)\right) \Longrightarrow & u_{+}=u_{M}(0) \\
\Longrightarrow & \Phi_{\text {thick }}=A(0) f\left(u_{M}(0), 0\right)=\Phi_{\text {lim }} .
\end{aligned}
$$

Then Lemma 4.2 gives CIII for the clarification zone.

$\Phi_{\text {lim }}=\Phi_{\text {thick }}<\Phi_{\mathrm{sb}}(0)$ : The inequality $\Phi_{\text {lim }}<\Phi_{\mathrm{sb}}(0)$ implies $f\left(u_{f}, 0\right)<$ $f\left(u_{m}(0), 0\right)$ with $u_{f}<u_{m}(0)$. The inequality $\Phi_{\text {thick }}<\Phi_{\mathrm{sb}}(0)$ gives $f\left(u_{+}, 0\right)<$ $f\left(u_{m}(0), 0\right)$, which implies $u_{+}<u_{m}(0)$. Since $s>f\left(u_{f}, 0\right)$, Lemma A.1 implies that $u_{-}=u_{+}=u_{f}$ and, finally, Lemma 4.3 gives TI.

$\Phi_{\text {lim }}=\Phi_{\text {thick }} \geq \Phi_{\mathrm{sb}}(0)$ : Hence, $f\left(u_{f}, 0\right) \geq f\left(u_{m}(0), 0\right)$ with $u_{f} \geq u_{m}(0)$. If $u_{m}(0) \leq u_{f} \leq u_{M}(0)$, then Lemma A.1 gives that either $u_{+}=u_{f}=u_{m}(0)$, which is impossible by Lemma A.2, or $u_{f}<u_{-}<u_{M}(0)=u_{+}$. Lemma 4.3 implies type TIIA (then $v(0) \leq \bar{v}$ ) or TIIB. If $u_{M}(0)<u_{f} \leq u_{\max }$, then Lemma A.1 gives $u_{-}=u_{+}=u_{f}$ and Lemma 4.3 implies type TIIA or B. Finally, if $v(D) \leq \bar{v}$, only TIIA is possible in both cases.

Acknowledgments. I would like to thank Dr. Gunnar Sparr at the Department and Dr. Michel Cohen de Lara, Cergrene, Paris, for reading the manuscript and providing constructive criticism.

\section{REFERENCES}

[1] F. M. Auzerais, R. Jackson, W. B. Russel, And W. F. Murphy, The transient settling of stable and flocculated dispersion, J. Fluid Mech., 221 (1990), pp. 613-639.

[2] N. G. Barton, C.-H. Li, AND J. Spencer, Control of a surface of discontinuity in continuous thickeners, J. Austral. Math. Soc. Ser. B, 33 (1992), pp. 269-289.

[3] M. C. Bustos And F. Concha, Boundary conditions for the continuous sedimentation of ideal suspensions, AIChE J., 38 (1992), pp. 1135-1138.

[4] M. C. Bustos And F. Concha, Settling velocities of particulate systems, 7. Kynch sedimentation process: Continuous thickening, Internat. J. Miner. Process., 34 (1992), pp. 33-51. 
[5] M. C. Bustos, F. Concha, And W. Wendland, Global weak solutions to the problem of continuous sedimentation of an ideal suspension, Math. Methods Appl. Sci., 13 (1990), pp. $1-22$.

[6] M. C. Bustos, F. Paiva, And W. Wendland, Control of continuous sedimentation as an initial and boundary value problem, Math. Methods Appl. Sci., 12 (1990), pp. 533-548.

[7] J.-P. Chancelier, M. C. DE LARA, AND F. PACARD, Analysis of a conservation pde with discontinuous flux: A model of settler, SIAM J. Appl. Math., 54 (1994), pp. 954-995.

[8] K. E. Davis, W. B. Russel, And W. J. Glantschnig, Settling suspensions of colloidal silica: Observations and X-ray measurements, J. Chem. Soc. Faraday Trans., 87 (1991), pp. $411-424$.

[9] S. DieHL, Continuous sedimentation of multi-component particles, Math. Methods Appl. Sci., to appear.

[10] S. DiEHL, On scalar conservation laws with point source and discontinuous flux function, SIAM J. Math. Anal., 26 (1995), pp. 1425-1451.

[11] S. DieHL, A conservation law with point source and discontinuous flux function modelling continuous sedimentation, SIAM J. Appl. Math., 56 (1996), pp. 388-419.

[12] S. DiEHL, Scalar conservation laws with discontinuous flux function: I. The viscous profile condition, Comm. Math. Phys., 176 (1996), pp. 23-44.

[13] S. Diehl And U. Jeppsson, A model of the settler coupled to the biological reactor, Wat. Res., to appear.

[14] S. Diehl, G. Sparr, And G. Olsson, Analytical and numerical description of the settling process in the activated sludge operation, in Instrumentation, Control and Automation of Water and Wastewater Treatment and Transport Systems, R. Briggs, ed., IAWPRC, Pergamon Press, Elmsford, NY, 1990, pp. 471-478.

[15] S. Diehl AND N.-O. WAllin, Scalar conservation laws with discontinuous flux function: II. On the stability of the viscous profiles, Comm. Math. Phys., 176 (1996), pp. 45-71.

[16] R. Dupont And M. Henze, Modelling of the secondary clarifier combined with the activated sludge model no. 1, Wat. Sci. Tech., 25 (1992), pp. 285-300.

[17] L. G. EKLund AND A. JERNQVIST, Experimental study of the dynamics of a vertical continuous thickener-I, Chem. Eng. Sci., 30 (1975), pp. 597-605.

[18] R. FONT, Calculation of compression zone height in continuous sedimentation, AIChE J., 36 (1990), pp. 3-12.

[19] R. Font And F. Ruiz, Simulation of batch and continuous thickening, Chem. Eng. Sci., 48 (1993), pp. 2039-2047.

[20] S. K. Godunov, A finite difference method for the numerical computations of discontinuous solutions of the equations of fluid dynamics, Mat. Sb., 47 (1959), pp. 271-306 (in Russian).

[21] L. HÄrtel AND H. J. PÖPEL, A dynamic secondary clarifier model including processes of sludge thickening, Wat. Sci. Tech., 25 (1992), pp. 267-284.

[22] A. M. IL'In AND O. A. OleiniK, Behaviour of the solutions of the Cauchy problem for certain quasilinear equations for unbounded increase of the time, Amer. Math. Soc. Transl. Ser. 2, 42 (1964), pp. 19-23.

[23] E. IsAacson AND B. TEMPle, Nonlinear resonance in systems of conservation laws, SIAM J. Appl. Math., 52 (1992), pp. 1260-1278.

[24] E. IsaAcson And B. Temple, Convergence of the $2 \times 2$ Godunov method for a general resonant nonlinear balance law, SIAM J. Appl. Math., 55 (1995), pp. 625-640.

[25] U. Jeppsson And S. DieHL, An evaluation of a dynamic model of the secondary clarifier, Wat. Sci. Tech., 34 (1996), pp. 19-26.

[26] U. Jeppsson AND S. DiEHL, On the modelling of the dynamic propagation of biological components in the secondary clarifier, Wat. Sci. Tech., 34 (1996), pp. 85-92.

[27] S. N. KRUžKov, First order quasilinear equations in several independent variables, Math. USSR-Sb., 10 (1970), pp. 217-243.

[28] G. J. KYNCH, A theory of sedimentation, Trans. Faraday Soc., 48 (1952), pp. 166-176.

[29] K. A. Landman, L. R. White, And R. Buscall, The continuous-flow gravity thickener: Steady state behaviour, AIChE J., 34 (1988), pp. 239-252.

[30] O. Lev, E. Rubin, And M. Sheintuch, Steady state analysis of a continuous clarifierthickener system, AIChE J., 32 (1986), pp. 1516-1525.

[31] T.-P. LiU, Nonlinear resonance for quasilinear hyperbolic equation, J. Math. Phys., 28 (1987), pp. 2593-2602.

[32] O. A. OLEINIK, Uniqueness and stability of the generalized solution of the Cauchy problem for a quasi-linear equation, Uspekhi Mat. Nauk, 14 (1959), pp. 165-170; Amer. Math. Soc. Transl. Ser. 2, 33 (1964), pp. 285-290.

[33] C. A. Petty, Continuous sedimentation of a suspension with a nonconvex flux law, Chem. Eng. Sci., 30 (1975), p. 1451. 
[34] M. Sheintuch, Steady state modeling of reactor-settler interaction, Wat. Res., 21 (1987), pp. 1463-1472.

[35] I. TAKÁcs, G. G. Patry, And D. Nolasco, A dynamic model of the clarification-thickening process, Wat. Res., 25 (1991), pp. 1263-1271.

[36] F. M. Tiller And W. Chen, Limiting operating conditions for continuous thickeners, Chem. Eng. Sci., 41 (1988), pp. 1695-1704.

[37] D. A. VACCARI AND C. G. UChrin, Modeling and simulation of compressive gravity thickening of activated sludge, J. Environ. Sci. Health., A24 (1989), pp. 645-674.

[38] Z. Z. Vitasovic, Continuous settler operation: A dynamic model, in Dynamic Modelling and Expert Systems in Wastewater Engineering, G. G. Patry and D. Chapman, eds., Lewis, Chelsea, MI, 1989, pp. 59-81. 